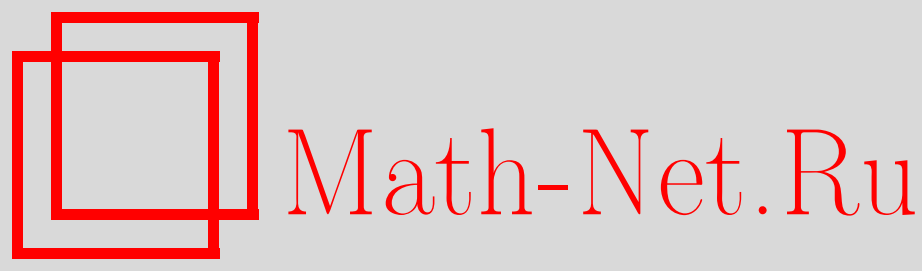

A. Pellegrinotti, V. Sidoravicius, M. E. Vares, Stationary state and diffusion for a charged particle in a one dimensional medium with lifetimes, Теория вероятн. и ее примен., 1999, том 44, выпуск 4, 796-825

DOI: https://doi.org/10.4213/tvp1066

Использование Общероссийского математического портала Math-Net.Ru подразумевает, что вы прочитали и согласны с пользовательским соглашением

http://www . mathnet.ru/rus/agreement

Параметры загрузки:

IP: 3.80 .181 .102

26 апреля 2023 г., 13:55:05

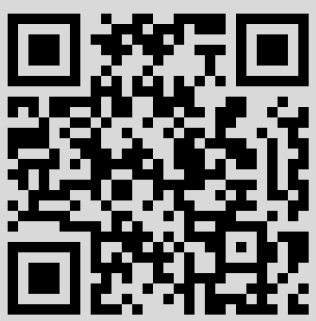




\title{
STATIONARY STATE AND DIFFUSION FOR A CHARGED PARTICLE IN A ONE DIMENSIONAL MEDIUM WITH LIFETIMES ${ }^{1)}$
}

\begin{abstract}
Изучается одномерная полубесконечная система одинаковых частиц с временем жизни, взаимодействующих с заряженной частицей (самой левой), на которую действует постоянная положительная сила $F$. Частицы взаимодействуют через упругие столкновения, в начальный момент все частицы находятся в покое, и расстояния между частицами - независимые, одинаково распределенные, положительные случайные величины. Каждая нейтральная частица имеет экспоненциальное время жизни, отсчет которого начинается, когда частица начинает двигаться. Времена жизни нейтральных частиц независимы и одинаково распределены. При подходящих условиях доказывается сильное кластерное свойство, сходимость к предельной мере для распределения системы, наблюдаемой с заряженной частицы, и центральная предельная теорема для движения заряженной частицы.
\end{abstract}

Ключевые слова и фразы: механическая система, стационарное состояние, кластерное свойство, центральная предельная теорема.

1. Introduction. The goal of this article is to contribute to the understanding of problems such as the motion of a test particle (t.p.) in a fluid, and the asymptotic behavior of the system, as seen from the t.p., which are basic in non-equilibrium statistical mechanics. Since the article of Harris in 1965 (see [12]), a large literature, both mathematical and physical, has been devoted to such problems. We refer to [22] for general discussion and further references. In particular, the analysis of stochastic dynamics led to several developments, and also provided good insight for the consideration of mechanical systems. (See second part of [22].) However, in spite of the big effort during the last three decades, there is at present no satisfactory

${ }^{*}$ Dipartimento di Matematica, Università degli Studi di Roma Tre, Roma, Italy; e-mail: pellegri@matrm3.mat.uniroma3.it

${ }^{* *}$ IMPA, Estrada Dona Castorina 110, Jardim Botanico, Rio de Janeiro, Brazil.

1) This work was partially supported by a PRONEX project (FINEP, Brasil) and by CNPq-CNR agreement. 
way to treat interacting Newtonian systems. Starting with Spitzer [21] and Holley [13], a class of systems which «a priori» have an invariant measure has been considered. The results of [21] were extended in [8], and convergence to a Wiener process has been proven in the equal masses case. When the mass of the t.p. goes to infinity, the one dimensional result of [13] was extended to dimension three, and convergence to an Ornstein-Uhlenbeck process has been obtained in [7]. In [23], Szász and Tóth studied the one dimensional Rayleigh gas, and for mass of the t.p. tending to infinity, a suitable class of scales is considered, under which they prove convergence to Brownian motion. Systems in which interaction is confined to a finite subsystem have been considered in [10] and [11].

During the last years several articles continuing this analysis have appeared. (See for instance [3], [9], [20], [24].) The validity of the Einstein relation has been treated in [4] and [15].

The model under our consideration consists of a system of infinitely many particles on the positive half line, which have equal masses, and are at rest at initial time. There is a charged particle (c.p.), initially located at the origin, on which a constant force is acting. No force acts on the other particles, called neutral particles (n.p.). The particles evolve by ellastic collisions.

A simplified version of this model was studied by Piasecki [17]; with some suitable restrictions on the minimum and the maximum for the interparticle distances, recollisions between the c.p. and n.p.'s were eliminated.

The next step was made in [2]. They consider a more complicated system, where initial interdistances are assumed to be an independent identically distributed (i.i.d.) sequence of random variables verifying:

(i) uniformly bounded away from zero and with exponentially decaying tails;

(ii) the minimal possible positive velocity given to a n.p. is bigger than the asymptotic drift of the c.p. in the corresponding Markov approximation (i.e., when each n.p. is annihilated after the first collision), as considered in [17].

Under these conditions, the validity of a strong cluster property is obtained, and it provides a key tool for proving existence of a limiting drift velocity for the charged particle. Under further technical condition convergence of the rescaled motion of the c.p. to a Wiener process is also proved.

Our goal is to understand how to release the above conditions (i), (ii). Absence of minimal interparticle distance leads to the non-existence of a minimal positive velocity and we bring into consideration the concept of suitable «clusters» of n.p.'s providing a sequence of times at which all moving particles have some minimal velocity (Minimal Velocity Lemma). As for 
condition (ii), its simple elimination raises the very delicate problem of how to control recollisions, which may bring a long term memory (see detailed discussion in [20], [21]). One alternative possible way, which we develop here, is through the introduction of «lifetimes» for each neutral particle.

The idea of existence of lifetimes was already indirectly used before (see for instance [3] and [5]). The geometric restrictions and conditions on the velocity distributions for the free gas particles of their models lead to the appearance of uniformly bounded «lifetimes».

In our model the interparticle distances at initial time are taken to be i.i.d. positive r.v.'s with exponentially decaying tails and, for the last results, we also need some technical condition (condition (C) below); moreover we assume n.p.'s to have lifetimes, which start counting as soon as the particle moves. These are taken to be i.i.d. exponential r.v.'s with some parameter $\nu>0$.

Under these conditions we obtain a cluster property, and as consequence we prove existence of an invariant measure for a suitable discrete dynamics, obtaining as well the asymptotic drift and an invariance principle for the charged particle. Representing the continuous dynamics as a «flow under a function» we have existence of an invariant measure for the continuous time dynamics as seen from the charged particle, and we prove convergence to this measure.

The article is organized as follows. In Section 2 we introduce the model and state the main results. In Section 3 we introduce the notions of «clusters» and prove the Minimal Velocity Lemma. This result holds also in the case of infinite lifetimes $(\nu=0)$. In Section 4 the strong cluster property is proven. In Section 5 we prove the existence of a stationary measure for the discrete dynamics which is obtained by looking at the system from the c.p., at the moments of collisions with standing particles (fresh collisions). Convergence to such measure is also proven. In Section 6 we prove an $\alpha$-mixing property which provides a Central Limit Theorem for the time of $n$th «fresh collision». We then prove the results for the continuous time dynamics.

2. Model and results. We consider a system of particles on the half line $\mathbf{R}_{+}=[0,+\infty)$, with non-negative velocities and lifetimes. We take as phase space of the system the set $X$ of all subsets $x \subseteq \mathbf{R}_{+} \times \mathbf{R}_{+} \times(0,+\infty]$ such that for each bounded interval $[0, b], \boldsymbol{x} \cap[0, b] \times \mathbf{R}_{+} \times(0,+\infty]$ is finite, and for each $q \geqslant 0, v \geqslant 0$ the set $x \cap\{(q, v)\} \times(0,+\infty]$ has at most one point. Each such element $x$ is thought as a marked point measure on $\mathbf{R}_{+} \times \mathbf{R}_{+}$, where the marks correspond to the lifetimes of the particles. We consider then $X$ with the topology for which a fundamental system of neighbourhoods of $x \in X$ is given by the sets

$$
G_{A, V, W}=\left\{x^{\prime} \in X: \#\left(x^{\prime} \cap(A \times V \times W)\right)=\#(x \cap(A \times V \times W))\right\},
$$


where $A, V(W)$ are relatively compact open sets of $\mathbf{R}_{+}((0,+\infty]$ respectively) with $x \cap \partial(A \times V \times W)=\varnothing$. Recall that with such topology $X$ is Polish space (as in [16]). Let $\mathscr{B}$ denote the Borel $\sigma$-field on $X$. It may be sometimes convenient to represent a configuration $x$ as a sequence $\left(q_{n}(x), v_{n}(x), \eta_{n}(x)\right)_{n \geqslant 0}$, where $q_{n}, v_{n}$, and $\eta_{n}$ will denote the position, velocity and lifetime of the $n$th particle, with the convention that $q_{n} \geqslant q_{n-1}$ and if $q_{n}=q_{n-1}$ then $v_{n}>v_{n-1}$, since $q_{n}=q_{n-1}, v_{n}=v_{n-1}$ will not occur in $X$.

The particles are all assumed to be pointlike, to have the same mass $m=1$, and to interact only through ellastic collisions. Moreover, the first particle has infinite lifetime and is subjected to a constant force $F>0$. Lifetime of each n.p. $p_{i}$ (which was initially located at $q_{i}$ ) starts to be discounted since the moment it gets positive velocity, that is, if at time $t$ particle $p_{i}$ has lifetime $\eta_{i}$ and velocity $v_{i}>0$ then it will disappear at time $t+\eta_{i}$.

The n.p.'s are thought as «pulses», so that we think that they exchange velocities only upon collision with charged particle. Since the n.p.'s are identical, except for the lifetimes, what we are doing amounts to exchanging lifetimes for «real particles».

At initial time $(t=0)$ all particles are at rest, i.e., $v_{i}=0 \forall i \geqslant 0$ and the c.p. is located at the origin $\left(q_{0}=0\right)$. The initial measure $\mu_{0}$ is then described by:

(A) the interparticle distances $\xi_{n}=q_{n}-q_{n-1}, n \geqslant 1$, are i.i.d. positive r.v.'s with an absolutely continuous distribution, and such that $\mathbf{E}_{\mu_{0}}\left(e^{\alpha \xi_{1}}\right)<+\infty$ for some $\alpha>0$;

(B) the lifetimes $\left(\eta_{n}\right)_{n \geqslant 1}$ are i.i.d. exponential r.v.'s with rate $\nu>0$ and independent of $\left(\xi_{n}\right)_{n \geqslant 1}$.

Under above conditions on $\mu_{0}$ we shall prove Theorem 1 and part (a) of Theorem 2. For Theorem 3 and part (b) of Theorem 2 we also need the following technical assumption:

(C) let $a_{0}=\inf \left\{a>0: \mu_{0}\left\{\xi_{1} \leqslant a\right\}>0\right\}$. Then: (i) if $a_{0}>\mathbf{E}_{\mu_{0}}\left(\xi_{1}\right) / 16$ we assume there exists $k \geqslant 1$ such that the $k$ th convolution of the conditional distribution $\Gamma$ of $\sqrt{2 \xi_{1} / F}$ given $\left[a_{0} \leqslant \xi_{1} \leqslant a_{0}^{\prime}\right]$, is absolutely continuous with a bounded density, whose Fourier transform is integrable, for some $a_{0}<a_{0}^{\prime}<4 a_{0}$; (ii) if $a_{0}<\mathbf{E}_{\mu_{0}}\left(\xi_{1}\right) / 16$ we assume there exist $a_{0}^{\prime}$ and $a_{0}^{\prime \prime}$ such that $0<a_{0}^{\prime \prime}<a_{0}^{\prime}<4 a_{0}^{\prime \prime} \wedge\left(\mathbf{E}_{\mu_{0}}\left(\xi_{1}\right) / 16\right)$ and that the analog to (i) holds with $a_{0}^{\prime \prime}$ replacing $a_{0}$ in the previous regularity condition.

$\mathrm{R}$ e $\mathrm{m}$ a $\mathrm{r} \mathrm{k} 1$. Such regularity assumption is an important tool for the validity of a local central limit theorem, used in Theorem 3 . For part (b) of Theorem 2, as the reader will check, it is enough that the above distribution $\Gamma$ is non-degenerated. One important class of examples would be when the distribution $\Gamma$ has itself a bounded density, in which case $k$ can be taken as 2 . 
$\mathrm{R}$ e $\mathrm{m}$ a $\mathrm{rk} 2$. The dynamics just described is well defined $\mu_{0}$-a.s. on $X$ and it is denoted by $\left(T_{t}\right)_{t \geqslant 0}$. This fact is also true for the dynamics with infinite lifetimes $(\nu=0)$, and its proof may be achieved through the following steps.

a) Reduction to finite subsystem with interdistances $\xi_{1}, \ldots, \xi_{n}$;

b) For each fixed $n$, and if $k \geqslant 1$, let

$$
M(k)=\left\{\left(\xi_{1}, \ldots, \xi_{n}\right): \text { the } k \text { th collision is multiple }\right\} .
$$

In [18] it has been proven that $\mu_{0}(M(k))=0$, for each $k$.

c) Having proven the absence (a.s.) of multiple collisions, for any given finite number of collisions, the argument of Lemma A.1 of [3] (the barycenter argument) gives us that the event of infinitely many collisions in finite time has measure zero (under $\mu_{0}$ ).

Later on, saying that $x$ is such that the dynamics is well defined, we refer to finitely many collisions in finite time, and no multiple collisions.

$\mathrm{D}$ efinition 1 . The position of the c.p. at time $t$ is denoted by $q_{0}(t, x)=q_{0}\left(T_{t} x\right)$.

The system, as seen from the c.p., is then described by the measure $\bar{\mu}_{t}=\mu_{0}\left(T_{t}^{0}\right)^{-1}$, where

$$
T_{t}^{0} x=S_{q_{0}(t, x)} T_{t} x, \quad S_{a} x=\{(q-a, v, \eta):(q, v, \eta) \in x\} .
$$

Similarly to [2] we consider the following discrete time dynamics.

$\mathrm{D}$ ef in ition 2. Given $x \in X$, let

$$
x_{0}=\{(q, v, \eta) \in x: v=0\}
$$

be the subset of the standing particles and $x_{m}=x \backslash x_{0}$. If $x \in X$ is such that dynamics is well defined then $q_{0}(t, x) \rightarrow+\infty$ as $t \rightarrow+\infty$ and we may define $t_{n}(x)$ through $q_{0}\left(t_{n}(x), x\right)=q_{n}\left(x_{0}\right), \tau_{n}(x):=t_{n}(x)-t_{n-1}(x)$, for each $n \geqslant 1,\left(t_{0}(x)=0\right)$, and $\mathbf{T}_{n} x=S_{q_{n}\left(x_{0}\right)}\left(T_{t_{n}(x)} x\right)$. Observe that if $X_{1}=\left\{x \in X: v_{0}=0\right\}$, and $\tau_{1}(x)=t_{1}(x)$ is well defined, then $\mathbf{T}_{1} x \in X_{1}$ and $\tau_{1}\left(\mathbf{T}_{1} x\right)=t_{2}(x)-t_{1}(x)$. Thus, whenever $t_{n}(x)$ is well defined we have

$$
\mathbf{T}_{n} x=\mathbf{T}_{1}^{n} x .
$$

For notational convenience let us set $\mathbf{T}=\mathbf{T}_{1}$.

In particular, the discrete evolution on $X_{1}$ is well defined ( $\mu_{0}$-a.s.).

$\mathrm{R} \mathrm{e} \mathrm{m}$ a r k 3. There is a short argument (due to C. Boldrighini) based on conservation laws which shows that if $\nu=0$ (infinite lifetimes) then ( $\mu_{0}$ a.s.):

$$
\sqrt{\frac{F}{2}} \sqrt{\mathbf{E}_{\mu_{0}} \xi_{1}} \leqslant \liminf _{t \rightarrow+\infty} \frac{q_{0}(t, x)}{t} \leqslant \limsup _{t \rightarrow+\infty} \frac{q_{0}(t, x)}{t} \leqslant \sqrt{\frac{F}{2}} \frac{\mathbf{E}_{\mu_{0}} \xi_{1}}{\mathbf{E}_{\mu_{0}} \sqrt{\xi_{1}}} .
$$


D e f i n ition 3 . We denote by $\mu_{n}$ the law of the system under the discrete dynamics, i.e.,

$$
\mu_{n}=\mu_{0}\left(\mathbf{T}_{n}\right)^{-1}=\mu_{0}\left(\mathbf{T}^{n}\right)^{-1}
$$

We may now state our main results:

Theorem 1. Assume $\nu>0$. There exists a probability measure on $X$ such that $\mu_{n} \rightarrow \mu$ weakly as $n \rightarrow+\infty$. In fact, $\mu_{n} \rightarrow \mu$ in variational distance, and exponentially fast in $n$.

Theorem 2. Assume $\nu>0$.

(a) There exists a positive constant $\bar{v}_{0}$ (depending on $\nu>0$ ) so that

$$
\lim _{t \rightarrow+\infty} \frac{q_{0}(t, x)}{t}=\bar{v}_{0} \quad\left(\mu_{0}-a . s .\right) \text {. }
$$

(b) If the distribution $\Gamma$ appearing in the condition (C) is nondegenerated, there exists a positive constant $\sigma$ such that the processes

$$
\left(\frac{q_{0}(u t)-\bar{v}_{0} u t}{\sigma \sqrt{u}}\right)_{0 \leqslant t \leqslant 1}
$$

converge in law, on $D([0,1], \mathbf{R})$, to a standard Brownian motion, as $u \rightarrow+\infty$.

Theorem 3. Assume $\nu>0$ and $\mu_{0}$ satisfies also condition $(C)$. If $\bar{\mu}_{t}$ denotes the law of $T_{t}^{0} x$ under $\mu_{0}$ (i.e., $\bar{\mu}_{t}(B)=\mu_{0}\left\{x: T_{t}^{0} x \in B\right\}$ ), then there exists a probability measure $\bar{\mu}$ on $X$ such that $\bar{\mu}_{t} \rightarrow \bar{\mu}$ weakly as $t \rightarrow+\infty$.

$\mathrm{N} \circ \mathrm{t}$ a $\mathrm{t}$ i o $\mathrm{n}$. For our initial configuration $x=\left(q_{i}, v_{i}, \eta_{i}\right)_{i \geqslant 0}$ with $v_{i} \equiv 0$, and according to our interpretation of n.p.'s as pulses crossing each other, if $i \geqslant 1$ we use $p_{i}$ to denote the pulse initially located at $q_{i}$, and let $q_{i}(t, x), v_{i}(t, x), \eta_{i}(t, x)$ be its position, velocity and remaining lifetime, at time $t$. Most of the time we will omit $x$ from notation, writing $q_{i}(t)$, $v_{i}(t), \eta_{i}(t)$.

In general, for $i \geqslant 1, q_{i}(t) \neq q_{i}\left(T_{t} x\right)$ due to different labelling.

3. Dynamics of clusters. Let us fix $0<d<\mathbf{E} \xi_{1} / 4$, from here on.

$\mathrm{D}$ e $\mathrm{f}$ i n ition 4 (d-conditional cluster). Let $\left(q_{n}(x)\right)_{n \geqslant 0}$ be the positions on the initial configuration $x$ (i.e., $q_{0}(x)=0, q_{n}(x)=\sum_{i=1}^{n} \xi_{i}$, if $n \geqslant 1$ ).

I. The first conditional cluster $\mathscr{C}_{1}^{x}$. Assume first $q_{1}>d$.

(a) The first particle $p_{1}$ will be the first particle of $\mathscr{C}_{1}^{x}$.

(b) Let $k \geqslant 1$ and suppose $p_{k} \in \mathscr{C}_{1}^{x}$. If $\left|q_{k+1}-q_{1}\right| \leqslant d+4(k-1) d$, we will say that $p_{k+1} \in \mathscr{C}_{1}^{x}$, otherwise we say that $p_{k}$ is the last particle of $\mathscr{C}_{1}^{x}$ and that $p_{k+1}$ is the first particle of the second cluster. In this case we will then say that $\mathscr{C}_{1}^{x}$ has cardinality $k$ and, when needed, use the notation $\mathscr{C}_{1}^{x}(k)$.

If $q_{1}<d$ condition $\mathrm{I}(\mathrm{b})$ is replaced by 
$\mathrm{I}\left(\mathrm{b}^{1}\right)$. Let $k \geqslant 1$ and assume $p_{k} \in \mathscr{C}_{1}^{x}$. If $\left|q_{k+1}\right|<d+4 k d$ we will say that $p_{k+1} \in \mathscr{C}_{1}^{x}$. Otherwise we say that $p_{k}$ is the last particle of $\mathscr{C}_{1}^{x}$ and $p_{k+1}$ is the first particle of $\mathscr{C}_{2}^{x}$.

II. The $n$th conditional cluster $\mathscr{C}_{n}^{x}$. Suppose we have already defined the $d$-conditional clusters $\mathscr{C}_{1}^{x}\left(m_{1}\right), \ldots, \mathscr{C}_{n-1}^{x}\left(m_{n-1}\right)$, of cardinality $m_{1}, \ldots, m_{n-1}$ respectively.

(a) $p_{m_{1}+\cdots+m_{n-1}+1}$ will be called the first particle of $\mathscr{C}_{n}^{x}$.

(b) Let $k \geqslant 1$, and suppose $p_{m_{1}+\cdots+m_{n-1}+k} \in \mathscr{C}_{n}^{x}$. If $\mid q_{m_{1}+\cdots+m_{n-1}+k+1}-$ $q_{m_{1}+\cdots+m_{n-1}+1} \mid \leqslant d+4(k-1) d$ we will say that $p_{m_{1}+\cdots+m_{n-1}+k+1} \in \mathscr{C}_{n}^{x}$; otherwise we say that $p_{m_{1}+\cdots+m_{n-1}+k}$ is the last particle of $\mathscr{C}_{n}^{x}$. In this case we say that $\mathscr{C}_{n}^{x}$ has cardinality $k$ and, if needed, we use the notation $\mathscr{C}_{n}^{x}(k)$.

$\mathrm{R} \mathrm{e} \mathrm{m} \mathrm{a} \mathrm{r} \mathrm{k} \mathrm{4.} \mathrm{Since} 4 d<\mathbf{E} \xi_{1}, \mu_{0}\left\{x: \mathscr{C}_{n}^{x}\right.$ is finite $\}=1$. In fact, there exist positive constants $c_{1}, \tilde{c}_{1}$ such that

$$
\mu_{0}\left\{x:\left|\mathscr{C}_{n}^{x}\right| \geqslant k\right\} \leqslant \widetilde{c}_{1} e^{-c_{1} k}
$$

for each $k \geqslant 1, n \geqslant 1$, as it follows from Chebyshev's exponential inequality.

$\mathrm{N}$ o t a $\mathrm{t}$ i o $\mathrm{n}$. Given a configuration $x \in X$ decomposed into $d$-conditional clusters $\left\{\mathscr{C}_{n}^{x}\left(m_{n}\right)\right\}_{n \geqslant 1}$, we set:

$$
\theta_{n}^{x}=q_{m_{1}+\cdots+m_{n-1}+1}+d+4\left(m_{n}-1\right) d \quad\left(m_{0}=0\right),
$$

which is called the endpoint of $\mathscr{C}_{n}^{x}$.

Clearly, at initial time, all particles of $\mathscr{C}_{n}^{x}\left(\mathscr{C}_{n+1}^{x}\right)$ are to the left (right, respectively) of $\theta_{n}^{x}$.

$\mathrm{Defin}$ ition 5 . Let $t_{n}^{*}$ be the time when c.p. reaches $\theta_{n}^{x}$, i.e., $\theta_{n}^{x} \equiv q_{0}\left(t_{n}^{*}(x), x\right)$.

From Remark 4, we immediately see that $\mu_{0}\left(t_{n}^{*}<+\infty\right)=1$, for any $\nu \geqslant 0$. We have now the following key estimate.

Lemma 1 (Minimal Velocity Lemma). Let $\nu \geqslant 0$. For $\mu_{0}$-almost all initial configurations, we have that at time $t_{n}^{*}$ all (alive) moving particles, including the charged particle, have velocity at least $v_{\min }=\sqrt{2 d F} / 4$.

For the proof of this lemma we first state, and prove the following

Lemma 2. Let $x \in X$ be a configuration with initially standing particles, for which dynamics is well defined. Let us set $k=\left|\mathscr{C}_{1}^{x}\right|$, as well as:

(a) $\psi_{1}=q_{1}$, and $\psi_{j}=q_{1}+d+4 d(j-2)$, for $2 \leqslant j \leqslant k$, if $q_{1} \geqslant d$;

(b) $\psi_{j}=d+4 d(j-1)$, for $1 \leqslant j \leqslant k$, if $q_{1}<d$.

Let now $u_{k}$ be the time when the charged particle «hits» $\psi_{k}$, i.e., $q_{0}\left(u_{k}(x), x\right)=\psi_{k}^{x}$. Then, at time $u_{k}$, all (alive) moving n.p.'s have velocity at least $v_{\min }$.

$\mathrm{Pr}$ o of. The proof will be by induction on $k$. Moreover we will consider only the case $q_{1} \geqslant d$, since otherwise, the modification is simple, with the proper change of the definition of cluster.

If $k=1$, the statement is trivial. Let us assume it to be true if $\left|\mathscr{C}_{1}^{x}\right| \leqslant$ $k-1$ but to be false for $\left|\mathscr{C}_{1}^{x}\right|=k$ with $k \geqslant 2$, and let us obtain a contradiction. 
Being the statement false we have a label $j_{1} \leqslant k$ so that $0<v_{j_{1}}\left(u_{k}\right)<v_{\min }$, and this implies $v_{j_{1}}\left(t_{j_{1}}\right)<v_{\min }$. Since at time $t_{j_{1}}$ n.p. $p_{j_{1}}$ collides with the c.p. and at time $u_{k}$ it is to the right of the c.p. we immediately get

$$
u_{k}-t_{j_{1}}>\frac{\psi_{k}-q_{j_{1}}}{v_{\min }}
$$

In this case we must have $2 \leqslant j_{1} \leqslant k$, since $v_{1}\left(t_{1}\right) \geqslant v_{\min }$. If $j_{1}=2$ we will immediately get a contradiction, since we would necessarily have $q_{2}<q_{1}+d / 16$ and the c.p. would have velocity less then $v_{\min }$ during time interval $\left[t_{1}, t_{2}\right]$, so that:

$$
u_{k}-t_{1}=u_{k}-t_{2}+t_{2}-t_{1}>\frac{d+4 d(k-2)}{v_{\min }}
$$

which contradicts the upper bound:

$$
u_{k}-t_{1} \leqslant \sqrt{\frac{2(k+1)}{F}(d+4 d(k-2))},
$$

provided by Lemma (cf. Appendix A).

If $2<j_{1}$ let $j_{2}^{\prime}<j_{1}$ be the label of the n.p. which last collided with the c.p. before $t_{j_{1}}$, and let $\tilde{t}_{j_{1}}$ be the time of this collision, so that during $\left[\tilde{t}_{j_{1}}, t_{j_{1}}\right]$ the c.p. has velocity less than $v_{\min }$, that implies

$$
t_{j_{1}}-\tilde{t}_{j_{1}}>\frac{q_{j_{1}}-q_{0}\left(\tilde{t}_{j_{1}}\right)}{v_{\min }}
$$

We have two possibilities. before

(a) If $0<v_{0}\left(\tilde{t}_{j_{1}}\right)<v_{\min }$ let $j_{2}=j_{2}^{\prime}$, so that $0<v_{j_{2}}\left(t_{j_{2}}\right)<v_{\min }$ and as

$$
\tilde{t}_{j_{1}}-t_{j_{2}}>\frac{q_{0}\left(\tilde{t}_{j_{1}}\right)-q_{j_{2}}}{v_{\min }} .
$$

If $j_{2}=2$ we will stop the procedure, getting a contradiction, just as above. If $j_{2}>2$ we continue the procedure, defining $j_{3}^{\prime}<j_{2}$ the label of n.p. with which the c.p. last collided before $t_{j_{2}}$, and $\tilde{t}_{j_{2}}$ the time of such collision, so that during $\left[\tilde{t}_{j_{2}}, t_{j_{2}}\right]$ the c.p. has velocity less than $v_{\min }$, implying as before

$$
t_{j_{2}}-\tilde{t}_{j_{2}}>\frac{q_{j_{2}}-q_{0}\left(\tilde{t}_{j_{2}}\right)}{v_{\min }}
$$

and repeating the procedure.

(b) If $v_{0}\left(\tilde{t}_{j_{1}}\right)=0$ (so that $\tilde{t}_{j_{1}}=t_{j_{2}^{\prime}}$ ) we ask if there is some moving n.p. at time $\tilde{t}_{j_{1}}$ with velocity less than $v_{\min }$. If answer is negative we stop the procedure and let $j_{2}=j_{2}^{\prime}$. If it is positive, we take $j_{2} \leqslant j_{2}^{\prime}-$ the label of one such n.p., so that $0<v_{j_{2}}\left(t_{j_{2}}\right)<v_{\min }$ and repeat argument for $j_{2}$ instead of $j_{1}$. 
In this way we will end up with a sequence $k \geqslant j_{1}>j_{2}>\cdots>j_{r} \geqslant 2$ so that:

(i) $j_{r}=2, v_{2}\left(t_{2}\right)<v_{\min }$, which will lead to (3.2), contradicting (3.3), or

(ii) $j_{r}>2$ and at time $t_{j_{r}}$ there is no moving n.p. with velocity less than $v_{\min }$.

If during time interval $\left(t_{j_{r}}, u_{k}\right]$ the c.p. does not recollide with $p_{1}, \ldots, p_{j_{r}}$ we consider the first cluster for the configuration of standing particles located at $q_{j_{r}+1}, \ldots, q_{k}$ and the c.p. located at $q_{j_{r}}$. Let $q_{j_{r}+1}, \ldots, q_{\ell}(\ell \leqslant k)$ be this cluster which has less than $k$ particles so that by induction hypothesis assumptions all such n.p. will reach $v_{\min }$ before the corresponding $\psi$, which is less or equal than $\psi_{\ell}$ (since $q_{j}<\psi_{j} \forall j$ ). Thus we get label $\ell+1>j_{r}$ and will repeat the argument for $p_{\ell+1}, p_{\ell+2}, \ldots, p_{k}$, until we arrive to $\psi_{k}$.

If there is recollision with $p_{1}, \ldots, p_{j_{r}}$, after $t_{j_{r}}$, let $\tilde{t}$ be the time of last such recollision, before $u_{k}$, and take $\ell$ so that $t_{\ell}<\tilde{t}<t_{\ell+1}$ for some $\ell \geqslant j_{r}$. In particular at $\tilde{t}$ all moving particles, including the c.p. have velocity at least $v_{\min }$, and we then repeat the argument for a cluster for the configuration of standing particles at $q_{\ell+1}, \ldots, q_{\tilde{\ell}}$ for some $\tilde{\ell} \geqslant \ell+1$. After a finite number of these «subcluster» considerations we would arrive at $u_{k}$ with all moving particles having velocity at least $v_{\min }$, contradicting our hypothesis.

As one can easily see the above argument works also in all cases $\nu \geqslant 0$.

$\mathrm{Pr}$ o of of $\mathrm{Lemma} 1$. For $\mathscr{C}_{1}^{x}$ the proof follows at once from Lemma 2 since if $\left|\mathscr{C}_{1}^{x}\right|=k(k \geqslant 1)$, then $\theta_{1}^{x}=\psi_{k+1} \geqslant \psi_{k}+d$, so that travelling from $\psi_{k}$ to $\theta_{1}^{x}$ the c.p. accelerates at least up to $v_{\min }$. We thus assume the statement proven for $\mathscr{C}_{1}^{x}, \ldots, \mathscr{C}_{n}^{x}$, and let us set $r, \ldots, r+k$ as being the labels of particles in $\mathscr{C}_{n+1}^{x}$. We have:

(a) $q_{r}>\theta_{n}^{x}$ and when the c.p. hits $\theta_{n}^{x}$ all moving particles from $\mathscr{C}_{1}^{x}, \ldots, \mathscr{C}_{n}^{x}$, as well as the c.p., have velocities at least $v_{\min }$; in particular $v_{r}\left(t_{r}\right) \geqslant v_{\min }$.

(b) $q_{r+1}-q_{r} \leqslant d, q_{r+j}-q_{r} \leqslant d+4 d(j-1)$ if $j \leqslant k ; q_{r+k+1}-q_{r}>d+4 d k$.

If the c.p. was not interacting with $p_{1}, \ldots, p_{r-1}$ during $\left[t, t_{r+k}\right]$ the result would follow from the first cluster case. If there is $j_{1} \in\{r, \ldots, r+k-1\}$ such that the c.p. meets these particles during some time interval $\left[t_{j_{1}}, t_{j_{1}+1}\right]$, then at this time all moving particles have velocity at least $v_{\min }$, and we would now repeat argument for $p_{j_{1}+1}, \ldots, p_{r+k}\left(j_{1}\right.$ may be the largest such label), proceeding just as at the end of the proof of Lemma 2.

We need to introduce the following

$\mathrm{D}$ e f i n i t i o n 6 (states $\mathscr{S}$ and $\mathscr{F}$ ). We say that the system is in state $\mathscr{S}$ at some time $t$ if there exists at least one moving particle (n.p. or c.p.) with velocity smaller than $v_{\min }$. Otherwise we say that system is in state $\mathscr{F}$ at this time.

Definition 7 . We say that n.p.'s $p_{\ell}, \ldots, p_{\ell+m-1}$ form a $d$-connected cluster of cardinality $m$ if:

(a) at time $t_{\ell}$ the system makes a transition from state $\mathscr{F}$ to state $\mathscr{S}$; 
(b) the system remains in state $\mathscr{S}$ up to $t_{\ell+m-1}$;

(c) before $t_{\ell+m}$ the system reaches state $\mathscr{F}$.

Since all particles are initially at rest, the system will immediately enter state $\mathscr{S}$; after some $\left(\mu_{0}\right.$-a.s. $)$ finite time it will achieve state $\mathscr{F}$; if in state $\mathscr{F}$, it will enter state $\mathscr{S}$ right after the c.p. meets the next standing particle, and so on. Indeed, as a consequence of Lemma 1 and equations (A.1) and (3.1), each of these periods will be a.s. finite. Moreover, from this we see that setting $\alpha_{1}=0$, and, for $k>1$,

$$
\begin{aligned}
w_{k} & =\inf \left\{t>\alpha_{k}: T_{t} x \text { is in state } \mathscr{F}\right\}, \\
\alpha_{k+1} & =\inf \left\{t>w_{k}: T_{t} x \text { is in state } \mathscr{S}\right\}
\end{aligned}
$$

(where we set inf $\varphi=+\infty$ ), we have

$$
\mu_{0}\left(\bigcap_{k \geqslant 1}\left\{\alpha_{k}<w_{k}<\alpha_{k+1}<\infty\right\}\right)=1,
$$

so that if $s_{k}=\left(w_{k}-\alpha_{k}\right) 1_{\left\{w_{k}-\alpha_{k}<+\infty\right\}}$ denotes the duration of $k$ th slow period, then each of these $s_{k}$ is a.s. finite.

Re mark 5. (a) From the estimate (3.1) for $n=1$ and equation (A.1) we easily get the existence of $a>0$ (independent of $\nu \geqslant 0$ ) such that

$$
\mathbf{E}_{\mu_{0}}\left(e^{a s_{1}}\right)<+\infty .
$$

(b) Conditioning on $\alpha_{k}=t_{m}$, using equation (3.1) and equation (A.1) for the configuration with origin shifted to $q_{m}$ we in fact have, by the same argument

$$
\sup _{m, k} \mathbf{E}_{\mu_{0}}\left(e^{a s_{k}} \mid \alpha_{k}=t_{m}\right)<+\infty
$$

for some $a>0$ independent of $\nu \geqslant 0$. In particular, there exists $a>0$ such that

$$
\sup _{k} \mathbf{E}_{\mu_{0}}\left(e^{a s_{k}}\right)<+\infty ; \quad \forall k \geqslant 1 .
$$

Lemma $3(\nu>0)$. Let us consider the n.p.'s which are alive at time $t_{k}\left(\alpha_{k}, w_{k}\right.$ resp.). Their remaining lifetimes are i.i.d. exponential r.v.'s with rate $\nu$.

$\mathrm{P}$ r o of. We first consider $t_{k}$, for this case details will be given. The other cases are similar.

Clearly, it is enough to look at moving particles, since at each time $t_{k}$ the system of standing particles is independent of the moving ones. For each $j$, the time $t_{j}$ depends on the initial configuration only through $\xi_{1}, \ldots, \xi_{j}$, $\eta_{1}, \ldots, \eta_{j-1}$, i.e.,

$$
t_{j}(x)=t_{j}\left(\eta_{1}, \ldots, \eta_{j-1}, \xi_{1}, \ldots, \xi_{j}\right)
$$


Let us fix $k \geqslant 1$ and let $\mathscr{G}_{k}$ and $\mathscr{D}_{k}$ be a partition of $\{1, \ldots, k\}$. We then define:

$$
A\left(k, \mathscr{G}_{k}, \mathscr{D}_{k}\right)=\bigcap_{j \in \mathscr{G}_{k}}\left\{\eta_{j}>t_{k}-t_{j}\right\} \bigcap \bigcap_{j \in \mathscr{D}_{k}}\left\{\eta_{j} \leqslant t_{k}-t_{j}\right\}
$$

i.e., the event that at time $t_{k}$, the $\mathscr{G}_{k}$-particles are alive and $\mathscr{D}_{k}$-particles are dead. Set

$$
\bar{\eta}_{j}= \begin{cases}\eta_{j} & \text { if } j \in \mathscr{D}_{k} \\ +\infty & \text { if } j \in \mathscr{G}_{k}\end{cases}
$$

and for $j \leqslant k$, set: $\bar{t}_{j}(x)=t_{j}\left(\bar{\eta}_{1}, \ldots, \bar{\eta}_{j-1}, \xi_{1}, \ldots, \xi_{j}\right)$,

$$
A_{\infty}\left(k, \mathscr{G}_{k}, \mathscr{D}_{k}\right)=\bigcap_{j \in \mathscr{G}_{k}}\left\{\eta_{j}>\bar{t}_{k}-\bar{t}_{j}\right\} \bigcap \bigcap_{j \in \mathscr{D}_{k}}\left\{\eta_{j} \leqslant \bar{t}_{k}-\bar{t}_{j}\right\} .
$$

It is easy to see that for a set of initial configurations $x$ of $\mu_{0}$-measure one (the set where dynamics is well defined) we have: if $x \in A\left(k, \mathscr{G}_{k}, \mathscr{D}_{k}\right) \cup$ $A_{\infty}\left(k, \mathscr{G}_{k}, \mathscr{D}_{k}\right)$ then $t_{j}(x)=\bar{t}_{j}(x)$ for each $j \leqslant k$, so that we may in fact conclude that

$$
A\left(k, \mathscr{G}_{k}, \mathscr{D}_{k}\right)=A_{\infty}\left(k, \mathscr{G}_{k}, \mathscr{D}_{k}\right) \quad\left(\mu_{0} \text {-a.s. }\right)
$$

and, if $k \geqslant 1, s_{1}, \ldots, s_{k}$ are positive,

$$
\begin{aligned}
\mu_{0} & \left\{\bigcap_{j \in \mathscr{G}_{k}}\left\{\eta_{j}>t_{k}-t_{j}+s_{j}\right\} \mid A\left(k, \mathscr{A}_{k}, \mathscr{D}_{k}\right)\right\} \\
& =\frac{\mu_{0}\left\{\bigcap_{j \in \mathscr{G}_{k}}\left\{\eta_{j}>t_{k}-t_{j}+s_{j}\right\} \cap \bigcap_{i \in \mathscr{D}_{k}}\left\{\eta_{i} \leqslant t_{k}-t_{i}\right\}\right\}}{\mu_{0}\left\{A\left(k, \mathscr{G}_{k}, \mathscr{D}_{k}\right)\right\}} \\
& =\frac{\mu_{0}\left\{\bigcap_{j \in \mathscr{G}_{k}}\left\{\eta_{j}>\bar{t}_{k}-\bar{t}_{j}+s_{j}\right\} \cap \bigcap_{i \in \mathscr{D}_{k}}\left\{\eta_{i} \leqslant \bar{t}_{k}-\bar{t}_{i}\right\}\right\}}{\mu_{0}\left\{\bigcap_{j \in \mathscr{G}_{k}}\left\{\eta_{j}>\bar{t}_{k}-\bar{t}_{j}\right\} \cap \bigcap_{i \in \mathscr{D}_{k}}\left\{\eta_{i} \leqslant \bar{t}_{k}-\bar{t}_{i}\right\}\right\}} \\
& =\frac{\mathbf{E}_{\mu_{0}}\left(\exp \left\{-\nu \sum_{j \in \mathscr{G}_{k}}\left(\bar{t}_{k}-\bar{t}_{j}+s_{j}\right)\right\} \prod_{i \in \mathscr{D}_{k}} \mathbf{1}_{\left(\eta_{i} \leqslant \bar{t}_{k}-\bar{t}_{i}\right)}\right)}{\mathbf{E}_{\mu_{0}}\left(\exp \left\{-\nu \sum_{j \in \mathscr{G}_{k}}\left(\bar{t}_{k}-\bar{t}_{j}\right) \prod_{i \in \mathscr{D}_{k}} \mathbf{1}_{\left(\eta_{i} \leqslant \bar{t}_{k}-\bar{t}_{i}\right)}\right\}\right)} \\
& =\exp \left(-\nu \sum_{j \in \mathscr{G}_{k}} s_{j}\right)
\end{aligned}
$$

where from the second to the last equality we have conditioned on the $\sigma$-field generated by $\left(\left(\eta_{i}\right)_{i \in \mathscr{D}_{k}}, \xi_{1}, \ldots, \xi_{k}\right)$.

$\mathrm{R}$ e $\mathrm{m}$ a $\mathrm{rk} 6$. The above argument shows indeed that, if we condition on the number of (alive) moving particles at time $t_{k}$, their remaining lifetimes are i.i.d. exponentially distributed, and independent of their positions and velocities.

4. Strong cluster property $(\nu>0)$.

Definition 8 . Let $x \in X$ be a configuration for which the dynamics is well defined and without multiple collisions. We then say that $k$ 
is a cluster index for $x$ if the c.p. will never collide after $t_{k}$ with those particles which are moving at time $t_{k}$, including $k$ th standing neutral particle.

Our main goal in this section is to prove the following

Theorem 4. Suppose $\nu>0$. Then

$\mu_{0}\{x: \exists i \leqslant n$ such that $i$ is a cluster index for $x\} \geqslant 1-c_{3} e^{-c_{4} n}$,

for suitable positive constants $c_{3}, c_{4}$.

We start by introducing the following procedure of counting the total number of moving n.p.'s, and for this let us fix $c>1 / \nu$. We fix a suitably large $N \in \mathbf{N}$ (to be determined later), and define:

(I) $\mathrm{F}$ i r s t cou $\mathrm{nt}$ i $\mathrm{g}$. If

$$
\begin{aligned}
& \sum_{j=1}^{k-1} s_{j}<c \ln 2 N \\
& \sum_{j=1}^{k} s_{j} \geqslant c \ln 2 N
\end{aligned}
$$

then we set $r_{1}=w_{k}$, which is called the time of the first counting. $\mathscr{N}(1)$ denotes the number of moving n.p.'s at time $r_{1}$.

(II) $(n+1)$ st c o u n t i n g. Assume $r_{1}, \ldots, r_{n}, \mathcal{N}(1), \ldots, \mathcal{N}(n)$ are already defined. If $r_{n}=w_{\ell}$ and

$$
\begin{aligned}
& \sum_{j=\ell+1}^{\ell+k-1} s_{j}<c \ln 2 N \\
& \sum_{j=\ell+1}^{\ell+k} s_{j} \geqslant c \ln 2 N
\end{aligned}
$$

then the time of the $(n+1)$ st counting will be $r_{n+1}=w_{\ell+k}$.

Notice that since each slow period has duration at least $\sqrt{d /(8 F)}$, the number of slow periods involved in each counting is at most $\mathscr{K}(N)=$ $[c \sqrt{8 F / d} \ln 2 N]+1([\cdot]$ denotes the integer part $)$. By $\mathcal{N}(n+1)$ we denote the number of moving n.p.'s at time $r_{n+1}$.

We need to control properly $\mathscr{N}(n)$. For this let $\mathscr{N}_{n}$ (old) be the number of moving n:p.'s at time $r_{n}$, which were already moving at time $r_{n-1}$. Set $\mathscr{N}_{n}($ new $)=\mathscr{N}(n)-\mathscr{N}_{n}$ (old).

Observe that if $L \geqslant 1$ :

$$
\begin{aligned}
& \left\{\mathscr{N}_{n+1}(\text { new })>L N\right\} \bigcap\left\{r_{n}=w_{\ell}\right\} \\
& \subseteq \bigcup_{j=\ell+1}^{\ell+\mathscr{K}(N)}\left\{\left|\tilde{\mathscr{C}}_{j}\right| \geqslant L N-\mathscr{K}(N)\right\} \bigcap\left\{r_{n}=w_{\ell}\right\}
\end{aligned}
$$

where $\tilde{\mathscr{C}}_{j}$ denotes the $j$ th $d$-connected cluster and $\left|\tilde{\mathscr{C}}_{j}\right|$ its cardinality. Indeed, the inequality (4.4) implies that at time $w_{\ell+k-1}$ the number of moving n.p.'s 
which were not moving at time $w_{\ell}$ is bounded by $\mathscr{K}(N)$. On the other hand if (4.5) holds and $\left|\tilde{\mathscr{C}}_{\ell+k}\right|<L N-\mathscr{K}(N)$ we will get $r_{n+1}=w_{\ell+k}$ and $\mathscr{N}_{n+1}$ (new) $<L N$.

If $t_{\varkappa-1}<r_{n}<t_{\varkappa}$ and since at this time system enters state $\mathscr{F}$, the motion of the c.p. during future slow periods does not depend on $\xi_{1}, \ldots, \xi_{\varkappa}, \eta_{1}, \ldots, \eta_{\varkappa}$. As a consequence the future connected clusters of $x$ coincide with the connected clusters of $x^{\varkappa}=\left(q_{j}^{(\varkappa)}, 0, \eta_{j}^{(\varkappa)}\right)_{j}$, where $q_{j}^{(\varkappa)}=$ $q_{\varkappa+j} ; \eta_{j}^{(\varkappa)}=\eta_{\varkappa+j}, j \geqslant 1$.

Thus, for any $d_{1}, \ldots, d_{n}, \ell, \varkappa$,

$$
\begin{aligned}
\mu_{0} & \left\{\mathscr{N}_{n+1}(\text { new })>L N \mid \mathscr{N}(1)=d_{1}, \ldots, \mathscr{N}(n)=d_{n},\right. \\
& \left.r_{n}=w_{\ell}, t_{\varkappa-1}<r_{n}<t_{\varkappa}\right\} \\
\leqslant & \mu_{0}\left(\bigcup_{j=1}^{\mathscr{K}(N)}\left\{\left|\tilde{\mathscr{C}}_{j}\left(x^{\varkappa}\right)\right| \geqslant L N-\mathscr{K}(N)\right\}\right) \\
\leqslant & \mathscr{K}(N) \mu_{0}\left\{\left|\mathscr{C}_{1}\right| \geqslant L N-\mathscr{K}(N)\right\},
\end{aligned}
$$

since the law of $\left|\tilde{\mathscr{C}}_{j}\left(x^{\varkappa}\right)\right|$ is stochastically smaller than that of $\left|\mathscr{C}_{1}\right|$.

From equations (3.1) and (4.6) there exists $N_{0}(d, c)$ such that if $N \geqslant N_{0}(d, c)$ and $\Sigma_{n}$ is the $\sigma$-field generated by $\mathscr{N}(1), \ldots, \mathcal{N}(n)$, we have

$$
\mu_{0}\left\{\mathscr{N}_{n+1}(\text { new })>L N \mid \Sigma_{n}\right\} \leqslant e^{-a L N}
$$

for some $a>0$ and all $n \geqslant 1, L \geqslant 1$. As for $\mathscr{N}_{n}$ (old), observe that always $r_{n+1}-r_{n} \geqslant c \ln 2 N$, and so, due to the loss of memory of the lifetimes

$$
\begin{aligned}
\mu_{0}\left\{\mathscr{N}_{n+1}(\text { old }) \leqslant \mathscr{N}(n)-2 N \mid \Sigma_{n}\right\} & \geqslant \mathbf{P}\left\{\max _{1 \leqslant i \leqslant 2 N} R_{i} \leqslant c \ln 2 N\right\} \\
& \stackrel{\text { def }}{=} p_{0}(2 N)
\end{aligned}
$$

$\left(\mu_{0}\right.$-a.s.) on $\{\mathscr{N}(n) \geqslant 2 N\}$, where $R_{1}, R_{2}, \ldots$ are i.i.d. exponential r.v.'s with rate $\nu$ on some space $(\Omega, \mathscr{A}, \mathbf{P})$, and where we are using Lemma 3.

In the same way on $\{\mathscr{N}(n)>N\}$ we have

$$
\mu_{0}\left\{\mathscr{N}_{n+1}(\text { old }) \leqslant \mathscr{N}(n)-N \mid \Sigma_{n}\right\} \geqslant p_{0}(N) \quad\left(\mu_{0} \text {-a.s. }\right) .
$$

Putting these estimates together we have:

$$
\begin{aligned}
\mu_{0}\left\{\mathscr{N}(n+1) \geqslant \mathscr{N}(n)+L N \mid \Sigma_{n}\right\} \leqslant & e^{-a L N} \quad \text { a.s., } \forall L \geqslant 1 ; \\
\mu_{0}\left\{\mathscr{N}(n+1) \leqslant \mathscr{N}(n)-N \mid \Sigma_{n}\right\} \geqslant & p_{0}(2 N)\left(1-e^{-a N}\right) \text { a.s. } \\
& \text { on }\{\mathcal{N}(n) \geqslant 2 N\} \\
\mu_{0}\left\{\mathcal{N}(n+1) \leqslant 2 N \mid \Sigma_{n}\right\} \geqslant & p_{0}(N)\left(1-e^{-a N}\right) \text { a.s. } \\
& \text { on }\{\mathscr{N}(n)<2 N\}
\end{aligned}
$$

if $N \geqslant N_{0}(d, c)$. 
Since $p_{0}(N)=\left(1-1 / N^{c \nu}\right)^{N}$, we immediately get that, provided $N$ is taken large enough, we can find a geometrically ergodic Markov chain $\left(Z_{n}\right)_{n}$, on $\{1,2, \ldots\}$, such that $\mathcal{N}(\cdot) \prec N Z(\cdot)+N$, in the stochastic sense on $\mathbb{N}^{N}$. The stochastic order is here defined in the usual way corresponding to the partial order $\prec$ on $\mathbf{N}^{\mathbf{N}}$, given by $\left(x_{n}\right)_{n} \prec\left(y_{n}\right)_{n}$ if and only if $x_{n} \leqslant y_{n}$ for each $n$. In fact, such chain may be taken as the random walk with transition probabilities:

$$
\begin{aligned}
p(i, i+j) & =e^{-\bar{a} j}\left(1-e^{-\bar{a}}\right) \quad \text { if } i, j \geqslant 1 \\
p(i, i-1) & =1-e^{-\bar{a}} \quad \text { if } i \geqslant 2 ; \\
p(1,1) & =1-e^{-\bar{a}},
\end{aligned}
$$

where $\bar{a}=\bar{a}(N)$ depends also on the other parameters and may be taken arbitrarily large, provided $N$ is also taken sufficiently large. This follows at once from inequalities (4.10) and the fact that $\lim _{N \rightarrow+\infty} p_{0}(N)=1$.

From here on $N$ is taken large enough as a function of $d\left(N \geqslant N_{1}(d, c)\right)$ so that the previous comparison works and the times between succesive returns to 1 for $\left(Z_{n}\right)_{n}$ have exponentially decaying tails. In particular, we may look at the following infinite subsequence of countings, called successful countings:

$$
n_{1}=\min \{n: \mathscr{N}(n) \leqslant 2 N\}, \quad n_{k+1}=\min \left\{n>n_{k}: \mathscr{N}(n) \leqslant 2 N\right\},
$$

for $k \geqslant 1$, and we may assume that there exist positive numbers $\widetilde{a}_{N}, \tilde{b}_{N}$, such that

$$
\mu_{0}\left\{n_{k} \geqslant k \widetilde{a}_{N}\right\} \leqslant e^{-k \widetilde{b}_{N}}
$$

for all $k \geqslant 1$.

We also let $m_{1}<m_{2}<\cdots$ be defined by $t_{m_{k}-1}<r_{n_{k}}<t_{m_{k}}$ for $k \geqslant 1$, where $t_{0}=0$.

$\mathrm{R}$ e m a r k 7. Observe that $m_{k} \leqslant \sum_{j=1}^{n_{k} \mathscr{K}(N)}\left|\tilde{\mathscr{C}}_{j}\right|+1$ and that the random variables $\left(\left|\tilde{\mathscr{C}}_{j}\right|\right)_{j \geqslant 1}$ are i.i.d., and from Lemma 1 we know that the law of $\left|\widetilde{\mathscr{C}}_{1}\right|$ is stochastically smaller then that of $\left|\mathscr{C}_{1}\right|$. Thus using (3.1) and the comparison of $\mathscr{N}(\cdot)$ with $\left(Z_{n}\right)_{n}$ we have

$$
\mu_{0}\left\{m_{k} \geqslant k a_{N}\right\} \leqslant e^{-k b_{N}}
$$

for suitable positive constants $a_{N}, b_{N}$ and for all $k \geqslant 1$. In particular,

$$
\lim \sup \frac{m_{k}}{k} \leqslant C_{N}<+\infty \quad\left(\mu_{0} \text {-a.s. }\right) \text {. }
$$

Let us now set:

$$
Y_{k}=\mathbf{1}_{\left[m_{k} \text { is a cluster index }\right]} .
$$

We then prove the following lemma, from which we immediately get the existence of a cluster index, provided $d$ is properly chosen. 
Lemma 4. We may choose $d<\mathbf{E}_{\mu_{0}}(\xi) / 4$, such that for each $N$ sufficiently large $\left(N \geqslant N_{1}(d, c)\right)$ there exist $\delta>0$ and $M \geqslant 1$ such that

$$
\begin{gathered}
\mu_{0}\left\{Y_{1}=1\right\} \geqslant \delta, \\
\mu_{0}\left\{Y_{k+M}=1 \mid Y_{1}, \ldots, Y_{k}\right\} \geqslant \delta \quad\left(\mu_{0} \text {-a.s. }\right)
\end{gathered}
$$

for each $k \geqslant 1$.

P r o of. Let $a_{0}=\inf \left\{a>0: \mu_{0}\left\{\xi_{1} \leqslant a\right\}>0\right\}$. We consider two cases.

C a s e 1. $a_{0} \geqslant \mathbf{E}_{\mu_{0}} \xi_{1} / 16$. In this case we may consider the event

$$
\left\{m_{1}=j\right\} \bigcap A_{j}^{N}
$$

where

$$
A_{j}^{N}=\bigcap_{i=1}^{[J \ln 2 N]+1}\left\{\xi_{j+i} \leqslant a_{0}^{\prime}\right\}
$$

$a_{0}<a_{0}^{\prime}<4 a_{0}$, and $J$ is the first integer such that $J \sqrt{d /(8 F)}>1 / \nu$. The events $\left\{m_{1}=j\right\}$ and $A_{j}^{N}$ are independent and

$$
\mu_{0}\left(A_{j}^{N}\right)=\left(\mu_{0}\left\{\xi_{1} \leqslant a_{0}^{\prime}\right\}\right)^{[J \ln 2 N]+1}=e^{-\alpha \ln 2 N} \text { for some } \alpha>0 .
$$

Obviously, if $x \in A_{j}^{N}$, then during the time interval $\left(t_{j}, t_{j+[J \ln 2 N]+1}\right)$ the c.p. cannot recollide with any n.p. with which it collides up to $t_{j}$. Thus, if all moving n.p.'s at time $t_{j}$ (including $p_{j}$ ) are annihilated by time $t_{j+[J \ln 2 N]+1}$, then $j$ is a cluster index. But

$$
t_{j+[J \ln 2 N]+1}-t_{j} \geqslant J \ln 2 N \sqrt{\frac{2 a_{0}}{F}}>J \sqrt{\frac{d}{8 F}} \ln 2 N
$$

and so we get, by the exponentiality of the remaining lifetime of alive n.p.'s at time $t_{j}$ :

$$
\begin{aligned}
\mu_{0}\left\{j \text { is a cluster index } \mid m_{1}=j\right\} & \geqslant\left(1-e^{-\nu J \sqrt{d /(8 F)} \ln 2 N}\right)^{2 N+1} \\
& \geqslant \delta>0
\end{aligned}
$$

where $\delta$ depends on $d$ and $N$. In the same way we get (4.14b) with $M=[J \ln 2 N]+1$, a function of $d$ and $N$.

$\mathrm{C}$ a s e 2. $a_{0}<\mathbf{E}_{\mu_{0}}(\xi) / 16$. Thus we may choose the parameter $d$ of our construction in such a way that $\mu_{0}\left\{\xi_{1} \leqslant d / 4\right\}>0$, and we consider the event

$$
\left\{m_{1}=j\right\} \bigcap A_{j}^{N} \bigcap B_{j}^{N}
$$


where

$$
\begin{aligned}
& A_{j}^{N}=\bigcap_{i=1}^{[J \ln 2 N]+1}\left\{\xi_{j+i} \leqslant \frac{d}{4}\right\}, \\
& B_{j}^{N}=\bigcap_{i=1}^{[J \ln 2 N]+1}\left\{\eta_{j+i}>J \sqrt{\frac{d}{8 F}} \ln 2 N\right\} \text {, }
\end{aligned}
$$

$J$ is as before. The events $\left\{m_{1}=j\right\}, A_{j}^{N}$ and $B_{j}^{N}$ are independent and if $M=[J \ln 2 N]+1$, then

$$
\mu_{0}\left(A_{j}^{N}(\xi) \bigcap B_{j}^{N}(\eta)\right)=\left(\mu_{0}\left\{\xi_{1} \leqslant \frac{d}{4}\right\}\right)^{M} e^{-\nu J M \sqrt{d /(8 F)} \ln 2 N} \geqslant e^{-\tilde{a}(\ln 2 N)^{2}}
$$

for some $\tilde{a}>0$. On $\left\{m_{1}=j\right\} \cap A_{j}^{N} \cap B_{j}^{N}$ we have that $p_{j}, \ldots, p_{j+[J \ln 2 N]+1}$ belong to the same connected cluster, the corresponding slow period will take time at least $J \sqrt{d /(8 F)} \ln 2 N$, and during this time the c.p. cannot recollide with any n.p. with which it collides up to $t_{j}$, since they have velocity at least $v_{\min }$. Thus, as in Case 1 we get (4.14a), and similarly, (4.14b).

$\mathrm{P}$ r o of of $\mathrm{Th}$ e or e $\mathrm{m} 4$. From Lemma 4 we get

$\mu_{0}\left\{\right.$ there is no cluster index among $\left.m_{1+M}, \ldots, m_{1+k M}\right\} \leqslant(1-\delta)^{k}$.

This together with (4.12b) gives us the proof of Theorem 4 .

$\mathrm{R}$ e $\mathrm{m}$ a $\mathrm{rk}$ 8. In Section 6 it will be convenient to look at cluster indices $k$ with the property that $k+1$ is also a cluster index at $x$. Analogously to [2], $k$ could be called «good cluster index». Let us observe that one way to obtain a good cluster index at a successful counting $m_{1}=j$ would be if we modify the definition of «trap» given in (4.15a) and (4.15b). Namely:

In Case 1 of the proof of Lemma 4 we replace the event $\left\{m_{1}=\right.$ $j\} \cap A_{j}^{N}$ by

$$
\left\{m_{1}=j\right\} \bigcap A_{j}^{N} \bigcap\left\{\eta_{j+1}<J \sqrt{\frac{d}{8 F}} \ln 2 N\right\}
$$

In Case 2 of that proof we replace the event $\left\{m_{1}=j\right\} \cap A_{j}^{N}$ by

$$
\left\{m_{1}=j\right\} \bigcap \widetilde{A}_{j}^{N} \bigcap \widetilde{B}_{j}^{N}
$$

where

$$
\begin{aligned}
& \widetilde{A}_{j}^{N}=\bigcap_{i=2}^{[J \ln 2 N]+1}\left\{\xi_{j+i} \leqslant \frac{d}{4}\right\} \bigcap\left\{a_{0}^{\prime \prime} \leqslant \xi_{j+1} \leqslant a_{0}^{\prime}\right\} \\
& \widetilde{B}_{j}^{N}=\bigcap_{i=2}^{[J \ln 2 N]+1}\left\{\eta_{j+i}>J \sqrt{\frac{d}{8 F}} \ln 2 N\right\} \bigcap\left\{\eta_{j+1}<J \sqrt{\frac{d}{8 F}} \ln 2 N\right\}
\end{aligned}
$$

with $a_{0}^{\prime \prime}, a_{0}^{\prime}$, according to condition (C) on $\mu_{0}$, and $4 a_{0}^{\prime}<d<\mathbf{E}_{\mu_{0}}\left(\xi_{1}\right) / 4$. 
With the same argument of Lemma 4 we see that for $N$ as previously chosen, there exist $\widetilde{\delta}>0$ and $M$ as in Lemma 4 so that if $\widetilde{Y}_{k}=\mathbf{1}_{\left[m_{k} \text { is a good cluster index }\right]}$ then

$$
\begin{gathered}
\mu_{0}\left\{\tilde{Y}_{1}=1\right\} \geqslant \widetilde{\delta} \\
\mu_{0}\left\{\widetilde{Y}_{k+M}=1 \mid \tilde{Y}_{1}, \ldots, \widetilde{Y}_{k}\right\} \geqslant \widetilde{\delta}
\end{gathered}
$$

which implies

$$
\begin{aligned}
& \mu_{0}\left\{\text { there is no good cluster index among } m_{1+M}, \ldots, m_{1+k M}\right\} \\
& \leqslant(1-\widetilde{\delta})^{k}
\end{aligned}
$$

and, due to (4.12)

$$
\mu_{0}\{x: x \text { has good cluster index } \leqslant n\} \geqslant 1-\widetilde{c}_{1} e^{-\widetilde{c}_{2} n}
$$

for suitable positive constants $\widetilde{c}_{1}, \widetilde{c}_{2}$.

$\mathrm{R} \mathrm{e} \mathrm{m} \mathrm{a} \mathrm{r} \mathrm{k} \mathrm{9.} \mathrm{Definition} 8$ corresponds to the natural concept of a cluster index. Nevertheless, for some arguments it is necessary to have a more specific definition, and we shall do this using the «trap» defined in the proof of Lemma 4. That is, we will say that $j$ is a cluster index for $x$, if: (a) at time $t_{j}$ there are at most $2 N$ moving n.p.'s all of them with velocity at least $v_{\min }$, and their remaining lifetimes are bounded by $J \sqrt{d /(8 F)} \ln 2 N$; (b) $x \in A_{j}^{N}$ (cf. (4.15b) in Case 1 of the proof of Lemma 4); (c) $x \in A_{j}^{N} \cap B_{j}^{N}$ cf. (4.17b) in Case 2 of the same proof).

From now on, when we say that $j$ is a cluster index we mean the above conditions. Similarly, for good cluster index, cf. equations (4.19).

5. Invariant measure for discrete dynamics. In this section we prove the convergence of the measures $\mu_{n}$, as $n \rightarrow+\infty$. We start by verifying the following

Lemma 5. The family $\left\{\mu_{n}\right\}_{n \geqslant 1}$ is tight.

$\mathrm{P} r$ o of. Just as in [2], it is enough to consider the moving particles, since the standing ones are distributed as $\mu_{0}$ and independent of the moving ones. Let then $M_{n}$ be the total number of (alive) moving particles at time $t_{n}$, i.e.,

$$
M_{n}=\#\left\{\mathbf{T}_{n} x \bigcap\left(\mathbf{R}_{+} \times(0,+\infty) \times(0,+\infty]\right)\right\} .
$$

We immediately see that

$$
M_{n} \leqslant 1+\sum_{i=1}^{n-1} \mathbf{1}_{\left[\eta_{i} \geqslant \sum_{j=i+1}^{n} \tilde{\xi}_{j}\right]},
$$

where

$$
\tilde{\xi}_{j}=\sqrt{\frac{2 \xi_{j}}{F}} .
$$


From this, it is easy to check that $\sup _{n} \mathbf{E}_{\mu_{0}}\left(M_{n}\right)<\infty$ implying the tightness. Indeed, from (5.2) taking the conditional expectations with respect to $\tilde{\xi}$ we have $\mathbf{E}_{\mu_{0}}\left(M_{n}\right) \leqslant 1+\sum_{j=1}^{n-1}(\varphi(\nu))^{j}$, where

$$
\varphi(u)=\mathbf{E}_{\mu_{0}}\left(e^{-u \widetilde{\xi_{1}}}\right), \quad \text { for } u>0 .
$$

$\mathrm{R}$ e $\mathrm{m}$ a r k 10. Though the previous trivial estimate suffices for the tightness, we will need later the following «exponential tightness».

Lemma 6. Let $M_{n}$ be defined by equation (5.1). Then there exists a $>0$ such that

$$
\sup _{n} \mathbf{E}_{\mu_{0}} e^{a M_{n}}<+\infty \text {. }
$$

$\mathrm{P}$ r o o f. Let us start with inequality (5.2). If $a>0$ we have:

$$
\begin{aligned}
\mathbf{E}_{\mu_{0}} \exp \left(a M_{n}\right) & \leqslant e^{a} \mathbf{E}_{\mu_{0}}\left(\prod_{i=1}^{n-1}\left(1+e^{a} \exp \left\{-\nu \sum_{j=i+1}^{n} \tilde{\xi}_{j}\right\}\right)\right) \\
& =e^{a} \mathbf{E}_{\mu_{0}}\left(1+\sum_{\substack{1 \leqslant j \leqslant n-1 \\
1 \leqslant i_{1}<\cdots<i_{j} \leqslant n-1}} e^{j a} \prod_{r=1}^{j} \exp \left(-\nu r \sum_{k=i_{r}+1}^{i_{r+1}} \tilde{\xi}_{k}\right)\right),
\end{aligned}
$$

where $i_{j+1}=n$.

Letting $\varphi(\cdot)$ be as in equation (5.4), we write

$$
\begin{aligned}
& \mathbf{E}_{\mu_{0}}\left(e^{a M_{n}}\right) \leqslant e^{a} \\
& +e^{a} \sum_{\substack{1 \leqslant j \leqslant n-1 \\
1 \leqslant i_{1}<\cdots<i_{j} \leqslant n-1}} e^{j a}(\varphi(j \nu))^{n-i_{j}}(\varphi((j-1) \nu))^{i_{j}-i_{j-1}} \ldots(\varphi(\nu))^{i_{2}-i_{1}} .
\end{aligned}
$$

We first assume $\varphi(\nu)<\frac{1}{2}$ and estimate the right-hand side of (5.6) by:

$$
\begin{aligned}
e^{a} & +e^{a} \sum_{\substack{1 \leqslant j \leqslant n-1 \\
1 \leqslant i_{1}<\cdots<i_{j} \leqslant n-1}} e^{j a}(\varphi(\nu))^{n-i_{1}} \\
& =e^{a}+e^{a} \sum_{1 \leqslant j \leqslant n-1} \sum_{1 \leqslant i \leqslant n-j} e^{j a}(\varphi(\nu))^{n-i}\left(\begin{array}{c}
n-1-i \\
j-1
\end{array}\right) \\
& =e^{a}+e^{2 a} \sum_{1 \leqslant i \leqslant n-1}(\varphi(\nu))^{n-i} \sum_{0 \leqslant j \leqslant n-i-1}\left(\begin{array}{c}
n-i-1 \\
j
\end{array}\right) e^{j a} \\
& =e^{a}+e^{2 a} \sum_{1 \leqslant i \leqslant n-1}(\varphi(\nu))^{n-i}\left(1+e^{a}\right)^{n-i-1} \\
& =e^{a}+e^{2 a} \varphi(\nu) \sum_{k=0}^{n-2}\left(\left(1+e^{a}\right) \varphi(\nu)\right)^{k}
\end{aligned}
$$

which will be bounded for $a>0$ such that $\left(1+e^{a}\right) \varphi(\nu)<1$. In the general case we take $m=m(\nu)$ so that $\varphi(m \nu)<\frac{1}{2}$ and repeat the above 
computations in a more careful way: we separate the terms corresponding to $j \leqslant m$ on the right-hand side of (5.6). For the sum of the others we proceed as before, but with $\varphi(m \nu)$ replacing $\varphi(\nu)$.

Our goal now is to prove Theorem 1. Proceeding as in [2], we may use a compactness argument, and prove the uniqueness of the weak limit points of $\left\{\mu_{n}\right\}$ by suitably coupling any such point with $\mu_{0}$.

Proposition 1. Let $\mu$ be a weak limiting point of $\left\{m^{-1} \sum_{i=1}^{m} \mu_{i}\right\}$. Then we can define a coupling $Q$ of $\mu$ and $\mu_{0}$, in such a way that there exists $\gamma>0$ such that

$$
Q\left\{\left(x, x^{\prime}\right): \mathbf{T}_{n} x \neq \mathbf{T}_{n} x^{\prime}\right\} \leqslant e^{-\gamma n} .
$$

$\mathrm{P}$ r o of. Let $\mu$ be as in the statement. Then, as in [2], $\mu=\mu_{0} \times \tilde{\mu}$, where $\tilde{\mu}$ is the distribution of moving particles, i.e.,

$$
\tilde{\mu}\left\{x \in X: \forall i \quad v_{i}(x)>0\right\}=1 .
$$

Now, we couple the measures $\mu$ and $\mu_{0}$ in the following way.

Let $x$ and $x^{\prime}$ be the corresponding configurations ( $\mu, \mu_{0}$, respectively). $x$ and $x^{\prime}$ will have the same standing particles, which will be distributed according to $\mu_{0}$, and we add - independently - the moving particles in configuration $x$, distributed according to $\tilde{\mu} . \quad x^{\prime}$ has no moving particles. That is,

$$
Q\left(d x, d x^{\prime}\right)=\delta_{\left(x_{0}^{\prime}=x_{0}\right)} \delta_{\left(x_{m}^{\prime}=\varnothing\right)} \mu_{0}\left(d x_{0}\right) \tilde{\mu}\left(d x_{m}\right),
$$

where, as before, $x_{0}\left(x_{m}\right)$ denotes the standing (moving, resp.) particles in $x$ $\left(x=x_{0} \cup x_{m}, x^{\prime}=x_{0}^{\prime}=x_{0}\right)$.

We need the following observations.

(a) There exists $\ell<+\infty(Q$-a.s. $)$ such that all moving particles in configurations $\mathbf{T}_{\ell} x$ and $\mathbf{T}_{\ell} x^{\prime}$ have velocity at least $v_{\min }$. Moreover, $\ell$ can be taken such that

$$
Q\left\{\left(x, x^{\prime}\right): \ell>n\right\} \leqslant e^{-\tilde{a} n}
$$

for some $\tilde{a}>0$ and all $n$ large enough. Indeed, let us first take $k \geqslant 8$ such that $(2 / k+1) 4 d<\mathbf{E}_{\mu_{0}}\left(\xi_{1}\right)$, and recall:

$$
\begin{aligned}
& Q\left\{\text { all initially moving n.p.'s in } x \text { die before } t_{[n / k]}(x)\right\} \\
& \quad \geqslant Q\left\{\text { all initially moving n.p.'s in } x \text { die before } L_{n / k}\left(x^{\prime}\right)\right\},
\end{aligned}
$$

where $L_{n / k}\left(x^{\prime}\right)=\sum_{i=1}^{[n / k]} \sqrt{2 \xi_{i}^{\prime} / F}$, and $\xi_{i}^{\prime}, i \geqslant 1$, denote the interdistances in $x^{\prime}$.

The right-hand side of (5.10) may be written as

$$
\int \mu_{0}\left(d x^{\prime}\right) \tilde{\mu}\left\{x_{m}: \# x_{m} \bigcap \mathbf{R}_{+} \times(0,+\infty) \times\left[L_{n / k}\left(x^{\prime}\right),+\infty\right]=0\right\} .
$$


Replacing first $\mathbf{R}_{+} \times(0,+\infty) \times\left[L_{n / k}\left(x^{\prime}\right),+\infty\right]$ by an increasing sequence of compact sets, using monotone convergence theorem, the fact that $\mu=$ $\tilde{\mu} \times \mu_{0}$ is the weak limit of some subsequence $\left\{\left(m_{j}\right)^{-1} \sum_{i=1}^{m_{j}} \mu_{i}\right\}$, as $j \rightarrow+\infty$, and also using Lemma 3 we get:

$$
\begin{aligned}
& \tilde{\mu}\left\{x_{m}: \# x_{m} \bigcap \mathbf{R}_{+} \times(0,+\infty) \times\left[L_{n / k}\left(x^{\prime}\right),+\infty\right]=0\right\} \\
& \quad \geqslant \liminf _{j \rightarrow+\infty}\left(1-\mathbf{E}_{\mu_{0}}\left(M_{j}\right) e^{-\nu L_{n / k}\left(x^{\prime}\right)}\right),
\end{aligned}
$$

where $M_{n}$ is defined in equation (5.1).

Since $\sup _{j} \mathbf{E}_{\mu_{0}}\left(M_{j}\right)<+\infty$ we get:

$Q\left\{\right.$ all initially moving n.p.'s in $x$ die before $\left.t_{[n / k]}(x)\right\} \geqslant 1-e^{-b n}$,

for suitable $b>0$ and all $n$ large enough. We now see how to get equation (5.9) out of this. If, at Lemma 1 some of the «fresh collisions» are replaced by collisions with moving particles, at those same positions, the result still holds, as it is easily seen from its proof. Thus if $x$ belongs to the event in (5.11), and $\xi_{[n / k]+1}+\cdots+\xi_{[n / 2]}>4 d[n / 2]$ then the evolution starting from $x$ will reach state $\mathscr{F}$ before $t_{[n / 2]}(x)$. But the event $\left\{\xi_{[n / k]+1}+\cdots+\xi_{[n / 2]}>4 d[n / 2]\right\}$ is independent of that of (5.11) and has probability converging to 1 exponentially fast as $n \rightarrow \infty$. We now take $\dot{l}-1$ as the index of the last particle in the $d$-conditional cluster in $x^{\prime}$ which contains $p_{[n / 2]}$, and we get (5.10).

(b) As a consequence of (a) we get that duration of slow periods in $x$ and $x^{\prime}$ will be exactly the same, after hitting $q_{\ell}$. (Of course $t_{\ell}(x) \neq t_{\ell}\left(x^{\prime}\right)$ in general.) That is, there will be $p, q$ such that for all $j \geqslant 1,\left|s_{p+j}(x)\right|=$ $\left|s_{q+j}\left(x^{\prime}\right)\right|$, and the motion of the c.p. during each slow period is equal for both evolutions.

(c) From the proof of Theorem 4 and Lemma 6 we can see that

$$
\inf _{j} \mu_{0}\left\{x^{\prime}: x^{\prime} \text { has cluster index in }\{j, j+1, \ldots, j+n\}\right\} \geqslant 1-e^{-\widetilde{b} n}
$$

for some $\widetilde{b}>0$ and $n$ large enough. This implies the existence of an infinite sequence of cluster indices.

Let us take one such cluster index $m, m>\ell$. From the construction, we know that all n.p.'s in $x^{\prime}$ which interact with the c.p. for the first time before $t_{m}\left(x^{\prime}\right)$ will be annihilated before the time $t_{m+M}\left(x^{\prime}\right)$, where $M=[J \ln 2 N]+1$ in the construction of Lemma 4. Recall that for this we have considered only slow periods, i.e., clock has been «stopped» during fast periods, so that for the evolution starting with $x$, all particles in $x_{0}$ which interact for the first time with the c.p. between $t_{\ell}(x)$ and $t_{m}(x)$, will be annihilated by time $t_{m+M}(x)$. If now $t_{+}$is the annihilation time of all n.p.'s in $x$ which move at $t_{\ell}(x)$, and $\ell^{\prime}(\cdot)=\min \left\{k \geqslant m(\cdot): t_{+}<t_{k}(x)\right\}$, 
from the above argument and equation (5.12) it follows that

$$
Q\left\{\ell^{\prime}>2 n\right\} \leqslant e^{-\tilde{c} n}
$$

for some positive constant $\tilde{c}$ and all $n$ large enough.

Now, if $K$ is any cluster index for $x^{\prime}$, which is larger than $\ell^{\prime}$, then $K$ will be a common cluster index. From equation (5.12) we may take $K$ so that

$$
Q\{K>3 n\} \leqslant e^{-h n}
$$

for some positive constant $h$ and all $n$ large enough. Now, equation (5.7) follows from (5.9), (5.13) and (5.14), since in this case the configurations $\mathbf{T}_{K+M}(\boldsymbol{x})$ and $\mathbf{T}_{K+M}\left(\boldsymbol{x}^{\prime}\right)$ coincide, as it follows from the construction of cluster index in Theorem 4.

Corollary 1. There exist $\gamma>0$ such that

$$
\sup _{k} \mu\left\{x: \mathbf{T}_{k+n}(x) \neq \mathbf{T}_{n}\left(x^{(k)}\right)\right\} \leqslant e^{-\gamma n}
$$

for all $n$ large enough, and where $x^{(k)}$ is the configuration with only standing particles, the c.p. at the origin, interdistances $\xi_{j}^{\prime}=\xi_{k+j}$, and lifetimes $\eta_{j}^{\prime}=\eta_{k+j}, j \geqslant 1$.

Proposition 2. There exist $\gamma_{0}>0$ such that

$$
\sup _{k} \mu_{0}\left\{x: \mathbf{T}_{k+n}(x) \neq \mathbf{T}_{n}\left(x^{(k)}\right)\right\} \leqslant e^{-\gamma_{0} n}
$$

for all $n$ sufficiently large, and where $x^{(k)}$ is as in Corollary 1.

$\mathrm{P}$ r oof. It is just the same as that of Proposition $1 x^{(k)}$ is the configuration of standing particles in $\mathbf{T}_{k} x$, which is independent of the moving ones. Moreover, the (alive) moving n.p.'s in $\mathbf{T}_{k} \boldsymbol{x}$ have lifetimes which are i.i.d. exponential with rate $\nu>0$, and due to Lemma 6 we may couple, as in Proposition 1, the evolutions starting from $x^{(k)}$ and from $\mathbf{T}_{k} x$, and the estimate does not depend on $k$.

$\mathrm{Pr}$ o of of $\mathrm{Th}$ e or e m 1. It follows at once from Lemma 5 and Proposition 1. In fact, the existence of $\mu$, a Cesaro weak limit point stationary for $\mathbf{T}$ follows from Lemma 5 . Thus equaiton (5.7) implies that

$$
\left\|\mu_{n}-\mu\right\|_{\mathrm{var}} \leqslant b_{1} e^{-\gamma n}
$$

for suitable positive constants $b_{1}, \gamma$, where $\|\cdot\|_{\text {var }}$ denotes total variation.

6. Mixing property and central limit theorem. Let $\mu$ be the limiting measure, given by Theorem 1 , so that $\mathbf{T}$ is a map on the space $\left(X_{1}, \mathscr{B}, \mu\right)$.

From equation (5.7) and Lemma 6 we have that the cardinal of moving particles has an exponential moment, under $\mu$. Moreover, this random 
variable is independent of the position of the first standing particle. Thus, from equation (A.1) (see Appendix A) it follows that

$$
\mathbf{E}_{\mu}\left(e^{a \tau_{1}}\right)<+\infty
$$

for some $a>0$. In particular, $\tau_{1}<+\infty$, ( $\mu$-a.s.) and we may then consider the stationary process $\left(\tau_{n}\right)_{n \geqslant 1}$ in $L_{1}\left(X_{1}, \mathscr{B}, \mu\right)$ defined by $\tau_{n}(x)=\tau_{1}\left(\mathbf{T}_{n-1} x\right)$ for $n \geqslant 2$.

We now prove the following

Lemma 7. The stationary sequence $\left(\tau_{n}\right)_{n \geqslant 1}$ on $L_{1}\left(X_{1}, \mathscr{B}, \mu\right)$ is strongly mixing, with exponentially decaying alpha coefficient, i.e.,

$$
\sup _{k \geqslant 1} \sup _{\substack{A \in \mathscr{M}_{1}^{k} \\ B \in \mathscr{M}_{k+n}^{\infty}}}|\mu(A \cap B)-\mu(A) \mu(B)| \leqslant c_{5} e^{-c_{6} n}
$$

for suitable positive constants $c_{5}, c_{6}$ and for all $n$, where $\mathscr{M}_{1}^{k}=$ $\sigma\left(\tau_{i}: 1 \leqslant i \leqslant k\right), \mathscr{M}_{k+n}^{\infty}=\sigma\left(\tau_{i}: k+n \leqslant i<+\infty\right)$.

$\mathrm{Pr}$ oof. This is an immediate consequence of Corollary 1 and Theorem 1 (or Proposition 1). Indeed, let $k \geqslant 1, n \geqslant 2$, and set

$$
C_{n}^{k}=\left\{x: \mathbf{T}_{k+n-1}(x)=\mathbf{T}_{n-1}\left(x^{(k)}\right)\right\},
$$

where $x^{(k)}$ is as in Corollary 1. By Corollary 1:

$$
\inf _{k} \mu\left(C_{n}^{k}\right) \geqslant 1-c_{7} e^{-\gamma n}
$$

for suitable positive constants $c_{7}, \gamma$.

Given $A \in \mathscr{M}_{1}^{k}, B \in \mathscr{M}_{k+n}^{+\infty}$, let $A^{\prime} \in \mathscr{B}\left(\mathbf{R}^{k}\right), B^{\prime} \in \mathscr{B}\left(\mathbf{R}^{\infty}\right)$ be such that $A=\left\{x:\left(\tau_{1}(x), \ldots, \tau_{k}(x)\right) \in A^{\prime}\right\}, \quad B=\left\{x:\left(\tau_{k+n}(x), \tau_{k+n+1}(x), \ldots\right) \in B^{\prime}\right\}$.

Observe that, from (6.3):

$$
A \bigcap B \bigcap C_{n}^{k}=A \bigcap\left\{x:\left(\tau_{n}\left(x^{(k)}\right), \tau_{n+1}\left(x^{(k)}\right), \ldots\right) \in B^{\prime}\right\} \bigcap C_{n}^{k} .
$$

But $A$ and $\left\{x:\left(\tau_{n}\left(x^{(k)}\right), \tau_{n+1}\left(x^{(k)}\right), \ldots\right) \in B^{\prime}\right\}$ are independent with

$$
\begin{aligned}
\mu\left\{x:\left(\tau_{n}\left(x^{(k)}\right), \tau_{n+1}\left(x^{(k)}\right), \ldots\right) \in B^{\prime}\right\} & =\mu_{n-1}\left\{x:\left(\tau_{1}(x), \tau_{2}(x), \ldots\right) \in B^{\prime}\right\} \\
& \stackrel{\text { def }}{=} \mu_{n-1}(\widetilde{B}),
\end{aligned}
$$

so that if $\widetilde{B}=\left\{x:\left(\tau_{1}(x), \tau_{2}(x), \ldots\right) \in B^{\prime}\right\}$ we have $\mid \mu(A \cap B)-$ $\mu(A) \mu_{n-1}(\widetilde{B}) \mid \leqslant 2 c_{7} e^{-\gamma n}$. Using now Theorem 1 (equation (5.15)) we conclude $(6.2)$, since $\mu(\widetilde{B})=\mu(B)$.

In order to prove Theorem 2 we may follow the same argument as in Theorem 2 in [2]. The crucial point which requires a different proof is the following 
Lemma 8. Let $\tau=\mathbf{E}_{\mu}\left(\tau_{1}\right)$ and $\bar{v}_{0}=\mathbf{E}_{\mu_{0}}\left(\xi_{1}\right) / \tau$ and consider the quantities

$$
\begin{aligned}
& \sigma^{2}=\mathbf{E}_{\mu}\left(\tau_{1}-\tau\right)^{2}+2 \sum_{k=2}^{\infty} \mathbf{E}_{\mu}\left(\left(\tau_{1}-\tau\right)\left(\tau_{k}-\tau\right)\right) \\
& \sigma^{\prime 2}=\mathbf{E}_{\mu}\left(\xi_{1}-\bar{v}_{0} \tau_{1}\right)^{2}+2 \sum_{k=2}^{\infty} \mathbf{E}_{\mu}\left(\left(\xi_{1}-\bar{v}_{0} \tau_{1}\right)\left(\xi_{k}-\bar{v}_{0} \tau_{k}\right)\right)
\end{aligned}
$$

which are well defined and finite. Then $\sigma^{2}>0$ and ${\sigma^{\prime 2}}^{2}>0$.

$\mathrm{P}$ r o of. The fact that $\sigma$ and $\sigma^{\prime}$ are well defined and finite follows from (6.1) and Lemma 7 (see, e.g., [14, Ch. 17]). Let $\mathscr{M}^{\prime}=\sigma\left(Y_{k}^{\prime}: k \geqslant 1\right)$, where

$$
Y_{k}^{\prime}=\mathbf{1}_{\{k \text { is a good cluster index }\}},
$$

and where we are using the «constructive definition», cf. Remark 9.

From (4.12b) and (4.21) we have the existence of a constant $p>0$ such that

$$
\lim \inf \left(\sum_{k=1}^{n} \frac{Y_{k}^{\prime}}{n}\right) \geqslant p \quad\left(\mu_{0} \text {-a.s. }\right) .
$$

Using now Proposition 1 the same is true under the measure $\mu$. So, for $\mu$-a.s. $x$ let $k_{1}<k_{2}<\cdots$ be such that $Y_{k_{i}}^{\prime}(x)=1$. It is not hard to see that, with our «construction» of good cluster indices:

(a) $\tau_{k_{1}+1}, \tau_{k_{2}+1}, \ldots$ are conditionally independent given $\mathscr{M}^{\prime}$, with a conditional distribution under $\mu$ (or $\mu_{0}$ ) given by $\Gamma$, as appearing in condition (C). By hypothesis (b) of Theorem 2 this distribution has a positive variance $\sigma_{0}^{2}$.

(b) The vectors $\left\{\tau_{k_{i}+2}, \ldots, \tau_{k_{i+1}}\right\}_{i \geqslant 1}$ are independent and for each $i$ the block is independent of $\tau_{k_{i}+1}$ under $\mu\left(\cdot \mid \mathscr{M}^{\prime}\right)$.

Thus, as in [2] we have

$$
\mathbf{E}_{\mu}\left(\left(t_{n}-\mathbf{E}_{\mu}\left(t_{n} \mid \mathscr{M}^{\prime}\right)\right)^{2} \mid \mathscr{M}^{\prime}\right) \geqslant \sigma_{0}^{2} \sum_{k=1}^{n} Y_{k}^{\prime} \quad(\mu \text {-a.s. })
$$

which then implies $\sigma^{2}>0$. The proof for $\sigma^{\prime}$ is similar.

P r o of of The or e m 2(P a r t (a)). We have

$$
\frac{q_{0}\left(t_{n}(x), x\right)}{t_{n}(x)}=\frac{q_{n}\left(x_{0}\right)}{t_{n}(x)}=\frac{n^{-1} \sum_{i=1}^{n} \xi_{i}}{n^{-1} \sum_{i=1}^{n} \tau_{i}}
$$

so that applying the strong law of large numbers on the numerator, and the ergodic theorem on the denominator (using here Lemma 7) we see that this expression converges a.s. under $\mu$ as $n \rightarrow+\infty$. By the coupling of Proposition 1 we also have a.s. convergence under $\mu_{0}$, and the limit is $\bar{v}_{0}=\mathbf{E}_{\mu_{0}}\left(\xi_{1}\right) / \mathbf{E}_{\mu}\left(\tau_{1}\right)$. 
If we now define

$$
\nu_{t}(x)=\max \left\{n: \sum_{i=1}^{n} \tau_{i} \leqslant t\right\}
$$

( $\nu_{t}$ is well defined and finite on a set of full measure for $\mu$, as well as for $\mu_{0}$ ), then

$$
\nu_{t} \rightarrow+\infty \quad \mu \text {-a.s. (as well as } \mu_{0} \text {-a.s.) as } t \rightarrow+\infty,
$$

and the conclusion follows by standard arguments.

Proof of $\mathrm{The}$ orem 2 ( $\mathrm{P}$ a $\mathrm{r}$ t (b)). The proof of (ii) in Theorem 2 of [2] works here provided we make a few considerations:

(a) Using (6.1), Lemmas 7 and 8 we may apply Theorem 18.5.3 of [14] to get that the $D[0,1]$-valued processes

$$
Z_{n}(\lambda, x)=\sum_{k=1}^{[n \lambda]} \frac{\tau_{k}-\tau}{\sigma \sqrt{n}}
$$

defined on $\left(X_{1}, \mathscr{B}, \mu\right)$, converge in law to the Wiener measure.

(b) Using Proposition 1 we see the above convergence holds also on $\left(X_{1}, \mathscr{B}, \mu_{0}\right)$.

(c) The same invariance principle holds for

$$
V_{n}(\lambda, x)=\sum_{k=1}^{[n \lambda]} \frac{\xi_{k}-\bar{v}_{0} \tau_{k}}{\sigma^{\prime} \sqrt{n}}
$$

for both $\mu$ or $\mu_{0}$.

(d) If $\nu_{t}$ is defined by equation (6.9) we also have $\nu_{t} / t \rightarrow 1 / \tau\left(\mu_{0}\right.$-a.s.) (see [1]). From this we have, just as in [2], that the processes

$$
\bar{V}_{n}(\lambda, x)=\sum_{k=1}^{\nu_{n \lambda}} \frac{\xi_{k}-\bar{v}_{0} \tau_{k}}{\sigma^{\prime} \sqrt{n}}
$$

tend in law to $(1 / \sqrt{\tau}) W(\lambda), W(\cdot)$ being the Wiener process.

(e) Going to continuous time and observing that

$$
\bar{V}_{t}(\lambda, x)-\frac{q_{0}(\lambda t, x)-\bar{v}_{0} \lambda t}{\sigma^{\prime} \sqrt{t}} \leqslant \frac{\xi_{\nu_{\lambda t}+1}+\bar{v}_{0} \tau_{\nu_{\lambda t}+1}}{\sigma^{\prime} \sqrt{t}}
$$

which tends to zero in $\mu_{0}$ probability the result follows, and $\bar{\sigma}=\sigma^{\prime} \sqrt{\tau}$.

$\mathrm{Pr}$ o of of The orem 3. Following standard procedure we may consider the flow under $\tau_{1}$ on $X_{1} \times \mathbf{R}$. For this, let $Y=\left\{(x, s) \in X_{1} \times \mathbf{R}\right.$ : $\left.0 \leqslant s<\tau_{1}(x)\right\}$, and set $T_{t}^{\prime}(x, s)=\left(\mathbf{T}_{\nu_{t+s}}, \theta_{t+s}\right)$, where $\nu_{t}$ was defined in equation (6.9), and $\theta_{t}=t-\sum_{i=1}^{\nu_{t}} \tau_{i}$.

It is known that the measure $\widehat{\mu}=\tau^{-1}(\mu \times \lambda)$ on $Y$ (where $\lambda$ denotes the Lebesgue measure) is invariant under $T_{t}^{\prime}$, and this yields an invariant 
measure $\bar{\mu}$ for the flow $T_{t}^{0}$ via $\bar{\mu}(B)=\widehat{\mu}\left\{(x, s) \in Y: T_{s}^{0} x \in B\right\}$ (cf. [2] and references therein).

As in [2] (proof of Theorem 3) we then see that Theorem 3 follows once we prove the following lemma (recall notation $\left.\mathbf{T}_{n}=\left(\mathbf{T}_{1}\right)^{n}\right)$.

Lemma 9. Let $\widehat{\mu}_{t}$ be the law of $\left(\mathbf{T}_{\nu_{t}}, \theta_{t}\right)$ under $\mu_{0}$. Then, as $t \rightarrow+\infty$ the measures $\hat{\mu}_{t}$ converge weakly (on $X_{1} \times \mathbf{R}_{+}$) to $\hat{\mu}$.

$\mathrm{R}$ e $\mathrm{m}$ a r k 11. Due to equation (5.15) we will in fact prove that for each bounded interval $I \subseteq(0,+\infty)$

$$
\lim _{t \rightarrow+\infty} \sup _{B \in \mathscr{B}}\left|\widehat{\mu}_{t}(B \times I)-\widehat{\mu}(B \times I)\right|=0 .
$$

As for the proof of Lemma 9 we may follow the same strategy as in Lemma 3.4 of [2]. The technical differences are due to: (i) we have proven only $\alpha$-mixing in Lemma 8; (ii) here the event $\{x: k$ is a cluster index for $x\}$ does not belong to $\sigma\left(\left(\xi_{j}, \eta_{j-1}\right): j \geqslant k\right)$, contrarily to [2]. One possibility would be to enlarge the $\sigma$-fields for which the (conditional) local central limit theorem (Lemma 10) is proven.

Thus, similarly to [2] we set

$$
\mathscr{B}_{n_{0}}^{n}=\sigma\left(\left(\xi_{k}, \eta_{k-1}\right): n_{0}+1 \leqslant k \leqslant n\right),
$$

where $n_{0}=n-\Delta n$ with $\Delta n=\left[b_{2} \ln n\right]$, for $n$ sufficiently large, and $b_{2}>0$ to be chosen later. Let also

$$
w_{n}=\frac{t_{n}-n \tau}{\sigma \sqrt{n}}, \quad w_{n}^{\prime}=\frac{t_{n}^{\prime}-n \tau}{\sigma \sqrt{n}}
$$

with $t_{n}^{\prime}$ being a suitable regularization of $t_{n}=\sum_{i=1}^{n} \tau_{i}$, i.e., $t_{n}^{\prime}=t_{n}+U$, with $U$ independent of all other variables, with a bounded density with compact support and whose Fourier transform is integrable. Let $P_{U}$ be the law of $U$, and $\mu_{0}^{\prime}=\mu_{0} \times P_{U}$.

Under such conditions one has the following

Lemma 10. For $n$ large enough there exists a set $G_{n} \in \mathscr{B}_{n_{0}}^{n}$ such that

$$
\begin{aligned}
& \mu_{0}\left(G_{n}\right) \geqslant 1-\frac{1}{n^{2}} \quad\left(\text { as well as } \mu\left(G_{n}\right) \geqslant 1-\frac{1}{n^{2}}\right), \\
& \sup _{x \in G_{n}} \sup _{y} \rho_{n}(y \mid x)-\frac{1}{\sqrt{2 \pi}} e^{-y^{2} / 2} \leqslant \frac{C}{n^{r}}
\end{aligned}
$$

for some positive constants $C, r$ independent of $n$, where $\rho_{n}(y \mid x)$ is the conditional density of $w_{n}^{\prime}$ given $\mathscr{B}_{n_{0}}^{n}$, under $\mu_{0}^{\prime}$.

The proof of this lemma is quite similar to that of Lemma A.1 of [2]; the different technical points are outlined in the Appendix.

$\mathrm{P}$ r o of of $\mathrm{L} \mathrm{e} \mathrm{m} \mathrm{m} \mathrm{a} 9$. From Proposition 2, and by taking $b_{2}$, in the definition of $n_{0}$, sufficiently large we have

$$
\mu_{0}\left\{x: \mathbf{T}_{n}(x) \neq \mathbf{T}_{\Delta n}\left(x^{\left(n_{0}\right)}\right)\right\} \leqslant \frac{1}{n^{2}}
$$


for $n$ large enough, where $x^{(i)}$ was defined in Corollary 1 .

Let us fix a Borel set $B \subseteq X_{1}$, and a bounded interval $I \subseteq \mathbf{R}_{+}$. Let us write $\nu_{t}^{\prime}, \theta_{t}^{\prime}$ for the regularized versions of $\nu_{t}, \theta_{t}$, i.e., $\nu_{t}^{\prime}=\max \left\{n: t_{n}^{\prime} \leqslant t\right\}$ and $\theta_{t}^{\prime}=t-t_{\nu_{t}^{\prime}}^{\prime}$. The r.v. $U$ used to regularized $t_{n}$ will be taken as $t$ dependent, with $U_{t}=t^{-2} \cdot U$, with $U$ as before. Thus, if $w_{t}$ is the Fourier transform of the density of $U_{t}$, then $\left\|w_{t}\right\|_{1}=t^{2}\|w\|_{1}$, where $\|\cdot\|_{1}$ is the $L_{1}$-norm on $(\mathbf{R}, d \lambda)$, and $w$ is the Fourier transform of the density of $U$.

First observe that

$$
\mu_{0}^{\prime}\left\{\theta_{t}^{\prime} \in I, \mathbf{T}_{\nu_{t}^{\prime}} \in B\right\}=\sum_{n=n_{1}(t)}^{n_{2}(t)} \mu_{0}^{\prime}\left\{\theta_{t}^{\prime} \in I, \mathbf{T}_{n} \in B, \nu_{t}^{\prime}=n\right\}+r(t),
$$

where $n_{1}(t)=[t / \tau]-[\sqrt{t} \ln t], n_{2}(t)=[t / \tau]+[\sqrt{t} \ln t]$, and $|r(t)| \leqslant \mu_{0}^{\prime}\left[\mid \nu_{t}^{\prime}-\right.$ $t / \tau \mid>\sqrt{t} \ln t] \longrightarrow 0, t \rightarrow+\infty$, as one easily checks.

Let now $n_{1}(t) \leqslant n \leqslant n_{2}(t)$, and if $G_{n}$ is given by Lemma 10 we may write, by (6.10)(i) and (6.11):

$$
\begin{aligned}
& \mu_{0}^{\prime}\left\{\theta_{t}^{\prime} \in I, \mathbf{T}_{n} \in B, \nu_{t}^{\prime}=n\right\} \\
& =\mu_{0}^{\prime}\left(\left\{\theta_{t}^{\prime} \in I, \mathbf{T}_{n} \in B, \nu_{t}^{\prime}=n, \mathbf{T}_{n}(x)=\mathbf{T}_{\Delta n}\left(x^{\left(n_{0}\right)}\right)\right\} \bigcap G_{n}\right) \\
& \quad+O\left(\frac{1}{n^{2}}\right)
\end{aligned}
$$

and if $I_{n}=\{y: t-n \tau-\sigma \sqrt{n} y \in I\}$, then

$$
\begin{aligned}
& \mu_{0}^{\prime}\left(\left\{\theta_{t}^{\prime} \in I, \mathbf{T}_{n} \in B, \nu_{t}^{\prime}=n, \mathbf{T}_{n}(x)=\mathbf{T}_{\Delta n}\left(x^{\left(n_{0}\right)}\right)\right\} \bigcap G_{n}\right) \\
& =\mu_{0}^{\prime}\left(\left\{w_{n}^{\prime} \in I_{n}, \tau_{\Delta n+1}\left(x^{\left(n_{0}\right)}\right)>t-n \tau-\sigma \sqrt{n} w_{n}^{\prime},\right.\right. \\
& \left.\left.\mathbf{T}_{\Delta n}\left(x^{\left(n_{0}\right)}\right) \in B\right\} \bigcap G_{n}\right)+O\left(\frac{1}{n^{2}}\right) \\
& =\int_{\left\{\mathbf{T}_{\Delta n}\left(x^{\left(n_{0}\right)}\right) \in B\right\} \cap G_{n}}\left[\int_{I_{n}} I_{\left\{\tau_{\Delta n+1}\left(x^{\left(n_{0}\right)}\right)>t-n \tau-\sigma \sqrt{n} y\right\}}\right. \\
& \left.\times \rho_{n}(y \mid x) d y\right] \mu_{0}(d x)+O\left(\frac{1}{n^{2}}\right)
\end{aligned}
$$

since $\left\{x: \mathbf{T}_{\Delta n}\left(x^{\left(n_{0}\right)}\right) \in B\right\} \cap G_{n} \in \mathscr{B}_{n_{0}}^{n+1}, \tau_{\Delta n+1}\left(x^{\left(n_{0}\right)}\right)$ is $\mathscr{B}_{n_{0}}^{n+1}$-measurable, and where we again used (6.11). From Lemma 10 the integral term on the right-hand side of equation (6.14) can be written as

$$
\int_{I_{n}} g(y) \mu_{0}\left\{x: \mathbf{T}_{\Delta n}\left(x^{\left(n_{0}\right)}\right) \in B, \tau_{\Delta n+1}\left(x^{\left(n_{0}\right)}\right)>t-n \tau-\sigma \sqrt{n} y\right\} d y+\varepsilon_{n}^{\prime},
$$

where $g(y)=(1 / \sqrt{2 \pi})^{-1} e^{-y^{2} / 2}$, and $\left|\varepsilon_{n}^{\prime}\right| \leqslant C / n^{1 / 2+r}$.

(Notice that in Lemma 10 we had $\mathscr{B}_{n_{0}}^{n}$ instead of $\mathscr{B}_{n_{0}}^{n+1}$, but obviously it works just the same, due to the independence of $\left.\left(\xi_{i}, \eta_{i-1}\right)_{i \geqslant 1}\right)$. 
From (6.12)-(6.15), it follows:

$$
\begin{aligned}
\mu_{0}^{\prime}\left\{\theta_{t}^{\prime} \in I, \mathbf{T}_{\nu_{t}^{\prime}} \in B\right\} & =\sum_{|k| \leqslant[\sqrt{t} \ln t]} \frac{1}{\sigma \sqrt{n_{1}(t)+k}} \\
\times \int_{I} d z & \left(g\left(\frac{t-n_{1}(t) \tau-k \tau-z}{\sigma \sqrt{n_{1}(t)+k}}\right)\right. \\
& \left.\times \mu_{0}\left\{\mathbf{T}_{\Delta\left(n_{1}(t)+k\right)} \in B, \tau_{\Delta\left(n_{1}(t)+k\right)+1}>z\right\}\right)+\varepsilon(t),
\end{aligned}
$$

where $\varepsilon(t) \rightarrow 0$ as $t \rightarrow+\infty$. As in Theorem 1 , this is easily seen to converge to $\tau^{-1} \int_{I} d z \mu\left(B \cap\left\{\tau_{1}>z\right\}\right)$.

It remains to pass from $\left(\mathbf{T}_{\nu_{t}^{\prime}}, \theta_{t}^{\prime}\right)$ to $\left(\mathbf{T}_{\nu_{t}}, \theta_{t}\right)$, but this is done as in [2] (equation (A.18) in [2]), using our condition (C).

Acknowledgements. The authors thank C. Boldrighini and E. Presutti for very fruitful discussions. The second and the third authors acknowledge warm hospitality at the Universities of Roma, and Camerino. The first author acknowledges warm hospitality at IMPA.

\section{Appendix A}

Lemma A.1. Let $x \in X_{1}$ be such that $T_{t} x$ is well defined and without multiple collisions. Suppose, moreover, that during the time interval $[0, t]$ the c.p. interacts with at most $k$ n.p.'s. Then

$$
t \leqslant \sqrt{\frac{2(k+1)}{F}\left(q_{0}(t)-q_{0}(0)\right)} .
$$

$\mathrm{P}$ r o of. For simplicity of writing let us assume the labels of particles with which the c.p. interacts during $[0, t]$ are $1, \ldots, k$. Moreover, let $v_{1}(0), \ldots, v_{k}(0)$ be their initial velocities $\left(v_{i}(0)\right) \geqslant 0$ by assumption), let $J \subseteq\{1, \ldots, k\}$ be the labels of those particles which died before $t$, and $s_{i}(i \in J)$ the times of their deaths. Without loss of generality we assume $v_{0}(0)=0$, and have

$$
\begin{aligned}
F t= & v_{0}(t)+\sum_{i \in J}\left(v_{i}\left(s_{i}\right)-v_{i}(0)\right)+\sum_{i \in\{1, \ldots, k\} \backslash J}\left(v_{i}(t)-v_{i}(0)\right) \\
q_{0}(t)-q_{0}(0)= & \frac{1}{2 F} \sum_{i \in\{0, \ldots, k\} \backslash J}\left(v_{i}(t)-v_{i}(0)\right)^{2}+\frac{1}{2 F} \sum_{i \in J}\left(v_{i}\left(s_{i}\right)-v_{i}(0)\right)^{2} \\
& +\sum_{i \in\{1, \ldots, k\} \backslash J} v_{i}(0) \frac{v_{i}(t)-v_{i}(0)}{F} \\
& +\sum_{i \in J} v_{i}(0) \frac{v_{i}\left(s_{i}\right)-v_{i}(0)}{F}
\end{aligned}
$$


as one may easily check since only finitely many collisions occur during $[0, t]$. In particular, since $v_{i}(t) \geqslant v_{i}(0)$ for $i=1, \ldots, k$, using Jensen's inequality (A.1) easily follows from (A.2) and (A.3).

$\mathrm{Pr}$ o of of $\mathrm{L}$ e m m a 10 . As already said, the proof essentially follows Lemma A.1 of [2]. Since there are a few technical differences, we outline the proof, emphasizing the points which require different argument or estimate.

Keeping the notation of [2], let $F_{n}(u \mid x)$, and $F_{n}^{\prime}(u \mid x)$ denote the characteristic function of the conditional distribution of $\widehat{t}_{n}$, and $\widehat{t}_{n}^{\prime}$, respectively, given $\mathscr{B}_{n_{0}}^{n}$. Also, let $f_{n}(u \mid x)=F_{n}(u /(\sigma \sqrt{n}) \mid x)$ and $f_{n}^{\prime}(u \mid x)=$ $F_{n}^{\prime}(u /(\sigma \sqrt{n}) \mid x)$ be the conditional characteristic functions of $w_{n}, w_{n}^{\prime}$.

The idea is to present a set $A_{n} \in \mathscr{B}_{n_{0}}^{n}$ as in the Lemma such that for some $\delta>0, \alpha>0, r>0$ :

$$
\begin{aligned}
\mid \int_{|u| \geqslant n^{\delta}}\left(f_{n}^{\prime}(u \mid x) d u \mid\right. & \leqslant\|w\|_{L_{1}} e^{-n^{\alpha}}, \\
\left|\int_{|u| \leqslant n^{\delta}}\left(f_{n}^{\prime}(u \mid x)-e^{-u^{2} / 2}\right) d u\right| & \leqslant n^{-r}
\end{aligned}
$$

for all $x \in A_{n}$, since this immediately implies the proof of the Lemma.

For (A.4) we need to exploit contribution of good cluster indices. More precisely, take $\frac{1}{2}<\beta<1$ and set

$$
D_{n}=\left\{x \in X: \exists \text { at least } n^{\beta} \text { good cluster indices in }\{1, \ldots, n\}\right\}
$$

(see Remarks 8 and 9.)

From the proof of Theorem 4, and Remarks 8 and 9 we see the existence of $\alpha>0$ such that $\mu_{0}\left(D_{n}^{c}\right) \leqslant e^{-4 n^{\alpha}}$ for all $n$ large enough, and we set $A_{n}^{\prime}=$ $\left\{x: \mu_{0}\left(D_{n_{0}}^{c} \mid \mathscr{B}_{n_{0}}^{n}\right)>e^{-2 n_{0}^{\alpha}}\right\}$ so that: (i) $A_{n}^{\prime} \in \mathscr{B}_{n_{0}}^{n} ;\left(\right.$ ii) $\mu\left(A_{n}^{\prime}\right)=\mu_{0}\left(A_{n}^{\prime}\right) \leqslant$ $e^{-2 n_{0}^{\alpha}}$ as one sees from the Chebyshev inequality. Conditioning first on the $\sigma$-field $\mathscr{B}_{n_{0}}^{\prime}=\mathscr{B}_{n_{0}}^{n} \vee \sigma\left(Y_{k}^{\prime}: 1 \leqslant k \leqslant n_{0}\right)$, where $Y_{k}^{\prime}=\mathbf{1}_{\{k \text { is a good cluster index }\}}$, and recalling the argument in the beginning of the proof of Lemma 7 plus our condition (C) we get (A.4), just as in [2], provided $\alpha<\beta+2 \delta-1$, which can be assumed if $1-\beta<2 \delta<1$.

As for (A.5) we need a more restrictive choice of $A_{n}$ : the idea is to approximate $f_{n}^{\prime}$ by the characteristic function of $w_{n_{0}}^{\prime}$ under $\mu_{0}-$ since this r.v. is independent of $\mathscr{A}_{n_{0}}^{n}-$ and for this we need to control $\hat{t}_{n}-\hat{t}_{n_{0}}$. Afterwards, due to Proposition 1 this is reduced to an estimate of the characteristic function of $w_{n_{0}}^{\prime}$ under $\mu$. At this point, one will have a problem of a stationary sequence of weakly dependent random variables. For this, set $\Delta t_{n}=t_{n}-t_{n_{0}}$, and observe that

$$
\Delta t_{n} \leqslant \sqrt{\frac{M_{n_{0}}+\Delta n+1}{2 F} \sum_{j=n_{0}+1}^{n} \xi_{j}} .
$$



we get:

But $M_{n_{0}}$ is independent of $\mathscr{B}_{n_{0}}^{n}$ and $\sup _{n} \mathbf{E}_{\mu_{0}}\left(M_{k}\right)<+\infty$, so that

$$
\mathbf{E}_{\mu_{0}}\left(\Delta t_{n} \mid \mathscr{B}_{n_{0}}^{n}\right) \leqslant C \Delta n+\frac{1}{2} \sum_{i=n_{0}+1}^{n} \xi_{i}
$$

Thus, if we set $A_{n}^{\prime \prime}=\left\{x: \mathbf{E}_{\mu_{0}}\left(\Delta t_{n} \mid \mathscr{B}_{n_{0}}^{n}\right) \geqslant(\Delta n)^{2}\right\}$ then: (i) $A_{n}^{\prime \prime} \in \mathscr{B}_{n_{0}}^{n}$; (ii) $\mu_{0}\left(A_{n}^{\prime \prime}\right) \leqslant 1 /\left(2 n^{2}\right)^{-1}$ for all $n$ large enough.

We then set $A_{n}=\left(A_{n}^{\prime}\right)^{c} \cap\left(A_{n}^{\prime \prime}\right)^{c}$, which will satisfy: (i) $A_{n} \in \mathscr{B}_{n_{0}}^{n}$; (ii) $\mu_{0}\left(A_{n}\right) \geqslant 1-n^{-2}$ for $n$ large enough.

To prove (A.5), and according to previous discussion, one sets:

$$
F_{n}^{0}(u)=\mathbf{E}_{\mu_{0}}\left(e^{i u \hat{t}_{n_{0}}}\right), \quad G(u)=\mathbf{E}_{\mu}\left(e^{i u \hat{t}_{n_{0}}}\right) .
$$

Thus if $n$ is large enough, then

$$
\left|F_{n}(u \mid x)-F_{n}^{0}(u)\right| \leqslant|i| \mathbf{E}_{\mu_{0}}\left(\left|\Delta t_{n}-\Delta n \tau\right| \mid \mathscr{A}_{n_{0}}^{n}\right) \leqslant|u|(\Delta n)^{2}
$$

for all $x \in A_{n}$.

Using Proposition 1 and that $\sup _{j} \mathbf{E}_{Q}\left(\tau_{j}(x)-\tau_{j}\left(x^{\prime}\right)\right)^{2}<+\infty$ we easily get:

$$
\left|F_{n}^{0}(u)-G(u)\right| \leqslant|u| \sum_{j=1}^{n_{0}} \mathbf{E}_{Q}\left[\mathbf{1}_{\left\{\mathbf{T}_{j} \neq \neq \mathbf{T}_{j} x^{\prime}\right\}} \mid \tau_{j}(x)-\tau_{j}\left(x^{\prime}\right)\right] \leqslant C^{\prime}|u|
$$

for suitable constant $C^{\prime}$.

Thus, (A.5) is reduced to an estimate for the characteristic function of $w_{n_{0}}$ under $\mu$. The classical proof (as in [2]; see also [14]) consists then of splitting $\left\{1, \ldots, n_{0}\right\}$ into «large» blocks $I_{k}$ of length $\left[n_{0}^{p}\right]$ and «small» blocks $J_{k}$ of length $\left[n_{0}^{q}\right]$ with $0<q<p<1$, and the «remainder». The estimates in our case are a little different from those of [2] since we have not proven a $\psi$-mixing property. Nevertheless, since our $\alpha$-mixing coefficient decays exponentially, and the variables $\tau_{k}$ have finite moments of all orders (see remark before Lemma 7), using Theorem 17.2.2 of [14] we get all the needed estimates, as in [2]. We omit full details, which can easily be reconstructed by the reader.

We just recall that the needed estimates are: (a) on the second moment of the sum of small blocks and remainder above; (b) on the third and fourth moment of one large block; (c) the fact that $\sup _{n}\left|\mathbf{E}_{\mu}\left(\hat{t}_{n}\right)^{2}-n \sigma^{2}\right|<+\infty$, which may all be proven as indicated.

\section{REFERENCES}

1. Биллиягсли П. Сходимость вероятностных мер. М.: Наука, 1977, 352 с.

2. Boldrighini C., Cosimi G., Frigio S., Nogueira A. Convergence to a stationary state and diffusion for a charged particle in a standing medium. - Probab. Theory Relat. Fields, 1989, v. 80, p. 481-500. 
3. Boldrighini C., Soloveitchik M. Drift and diffusion for a mechanical system. Probab. Theory Relat. Fields, 1995, v. 103, p. 349-379.

4. Boldrighini C., Soloveitchik M. On the Einstein Relations for a Mechanical System. - Probab. Theory Relat. Fields, 1997, v. 107, № 4, p. 493-515.

5. Calderoni P., Durr D. The Smoluchowsky limit for a simple mechanical model. J. Statist. Phys., 1989, v. 5, № 3/4, p. 695-738.

6. Cornfeld I.P., Fomin S.V., Sinai Ya.G. Ergodic Theory. Berlin-Heidelberg: Springer-Verlag, 1982.

7. Dürr D., Goldstein S., Lebowitz J.L. A mechanical model of Brownian motion. Comm. Math. Phys., 1981, v. 78, p. 507-530.

8. Dürr D., Goldstein S., Lebowitz J. L. Asymptotics of particle trajectories in infinite one-dimensional systems with collisions. - Comm. Pure Appl. Math., 1985, v. 38, p. 573-597.

9. Erdös L., Tuyen D. Q. Central limit theorems for the one-dimensional Rayleigh gas with semipermeable barriers. - Comm. Math. Phys., 1992, v. 143, p. 451-466.

10. Goldstein S., Lebowitz J. L., Ravishankar K. Ergodic properties of a system in contact with a heat bath: a one dimensional model. - Comm. Math. Phys., 1982, v. 85, № 3, p. 419-427.

11. Goldstein S., Lebowitz J. L., Ravishankar K. Approach to equilibrium in models of a system in contact with a heat bath. - J. Statist. Phys., 1986, v. 43, № 12, p. 303-315.

12. Harris T.E. Diffusion with collisions between particles. - J. Appl. Probab., 1965, v. 2, p. 323-338.

13. Holley $R$. The motion of a heavy particle in an infinite one dimensional gas of hard spheres. - Z. Wahrscheinlichkeitstheor. verw. Geb., 1971, v. 17, p. 181-219.

14. Ибрагимов И. А., Линник Ю. В. Независимые и стапионарно связанные величины. М.: Наука, 1965, 524 с.

15. Lebowitz J. L., Rost H. The Einstein relation for the displacement of a test particle in a random environment. - Stochastic Process. Appl., 1994, v. 54, № 2, p. 183-196.

16. Lenard $A$. States of classical statistical mechanics of infinitely many particles. Arch. Rat. Mec. Anal., 1975, v. 59, p. 219-241.

17. Piasecki J. Approach to field-induced stationary state in a gas of hard rods. J. Statist. Phys., 1983, v. 30, p. 201-209.

18. Sidoravicius V., Triolo L., Vares M.E. On the forced motion of a heavy particle in a random medium II. Existence of dynamics. - Markov Process. Relat. Fields, 1988, v. 4, № 3, p. 423-443.

19. Sinai Ya. G., Soloveitchik M. One dimensional classical massive particle in the ideal gas. - Comm. Math. Phys., 1986, v. 104, p. 423-443.

20. Soloveitchik $M$. Conservative dynamical system in a polyhedral angle. Existence of dynamics. - Non-linearity, 1995, v. 8, p. 367-378.

21. Spitzer $F$. Uniform motion with elastic collisions of an infinite particle system. J. Math. Mech., 1968, v. 18, p. 973-989.

22. Spohn H. Large Scale Dynamics of Interacting Particles. Berlin: Springer-Verlag, 1991, 342 p.

23. Szász D., Tóth B. Dynamical theory of Brownian motion for the Rayleigh gas. J. Statist. Phys., 1987, v. 47, p. 681-693.

24. Uchiyama $K$. Scaling limit for a mechanical system of interacting particles. - Comm. Math. Phys., 1996, v. 177, p. 103-128. 